\title{
4. Zur forschungspraktischen Herangehensweise
}

Die Unterscheidung unterschiedlicher Typen von Bürgerwehren in Deutschland zeigt, wie divers das Phänomen ist. Die Unterschiede zwischen den einzelnen Gruppen manifestieren sich insbesondere im Auftreten der Bürgerwehren im öffentlichen Raum, sie zeigen sich in ihrem Umgang mit der Macht, die ihnen ihre Rolle verleiht. Aber auch auf subtileren Ebenen werden die Unterschiede zwischen den Bürgerwehren im Akt der Patrouille sichtbar. Ihr Habitus, das Verständnis ihrer Aufgabe, Sicherheit zu schaffen, ihre Kleidung, auf welche Weise Hilfsmittel eingesetzt werden, wie Passant*innen auf sie reagieren - all diese Merkmale charakterisieren die Bürgerwehren. Diese Charakteristika lassen sich aber nur erfassen, wenn der Moment betrachtet wird, in dem die Bürger*innen zu Mitgliedern der Bürgerwehr werden. In dem Moment, in dem die Bürgerwehr als solche im öffentlichen Raum auftritt, schlüpfen die Mitglieder in ihre Rolle. Sie verhalten sich gemäß den Attributen, die sie dieser Rolle zuschreiben.

Bürgerwehren produzieren kaum schriftliche Quellen. Es existieren zwar Zeitungsartikel über ihr Handeln, aber die Darstellung ist vom Blick der Journalist*innen und von der Auswahl der Zitate abhängig. Zwar sind einige Bürgerwehren in sozialen Medien aktiv, doch lassen Haltungen, die in SocialMedia-Posts ausgedrückt werden, nicht ohne Weiteres auf ein Agieren im öffentlichen Raum schließen. Die Gründung von Bürgerwehren als Versicherheitlichungsprozess zu untersuchen, muss diese Studie also die Mitglieder der Bürgerwehr, die securitizing actors, in ihrer Rolle als Bürgerwehr betrachten.

Gleichzeitig ist im Versicherheitlichungsakt Sprache das zentrale Mittel, mit dem Versicherheitlichung erreicht wird. Durch eine breitere kommunikative Handlung (performative action) wird der Versicherheitlichungsprozess erfolgreich. Daher wird für diese Studie eine ethnographische Methode gewählt, die einen direkten Zugang zur Erfahrung der Bürgerwehr in der Aus- 
übung ihrer Macht ermöglicht und gleichzeitig die Legitimationen, die die Bürgerwehren für ihr Handeln anführen, erfasst.

Ethnografische Forschung fußt auf der Annahme, dass Sozialität in Situationen stattfindet, und dass die agierenden Menschen in diesen Situationen einen privilegierten Zugang zu den sozialen Relevanten der Situation haben. Will man also die Strukturen beschreiben, die das Soziale ordnen, muss man möglichst nah heran an diesen privilegierten Zugang. ${ }^{1}$

Die Feldforschung als methodischer Zuschnitt der Ethnografie setzt diese Forderung um. Feldforschung ist das persönliche Aufsuchen von Lebensräumen. Bewusst wird hier in Abgrenzung zu künstlich hergestellten experimentellen Situationen die soziale Alltagssituation gewählt. Das Verhalten der sozialen Gruppe soll so authentisch wie möglich bleiben und durch die Beobachtungssituation möglichst wenig verändert werden. ${ }^{2}$

Was jedoch für diese Studie darüber hinaus von Interesse ist, sind die Positionierungen der Bürgerwehr zum Themenbereich Sicherheit. Die Selbstverortung der Bürgerwehr als Sicherheitsakteur, die Argumentationen für die Notwendigkeit des Agierens im öffentlichen Raum, die Deutungen der politischen Entwicklungen - diese Bereiche zu betrachten ist wichtig, um das Verhältnis der Bürgerwehren zum Staat untersuchen zu können. Daher wird in der vorliegenden Studie das aus der teilnehmenden Beobachtung gewonnene Material durch Datenmaterial ergänzt, das aus Interviews im Feld gewonnen wurde. Hier sind die Mitglieder der Bürgerwehr emotional in ihrer Rolle als Bürgerwehr und sprechen spontan aus ihr heraus.

Interviews legen den Fokus auf Deutungen und Argumentationen. Hier lässt sich auf einer abstrakteren verbalisierten Ebene ergänzen, was in der teilnehmenden Beobachtung einer Patrouille über die politische Selbstverortung herausgefunden wurde. Zudem können die Mitglieder der Bürgerwehr von früheren Patrouillen erzählen, und es lassen sich Motive für die Gründung der Bürgerwehr abfragen. Die Interviews werden als Gruppeninterviews im Feld geführt, weil alle Mitglieder der Bürgerwehr relevant für die Untersuchung sind und ihre Anzahl überschaubar ist. So können die unterschiedlichen Perspektiven innerhalb der Bürgerwehr abgebildet werden und Gruppenprozesse erfasst werden.

1 Vgl. Breidenstein, Ceorg et al. (2013): Ethnographie. Eine Praxis der Feldforschung. Konstanz: UVK. S. 41.

2 Vgl. ebd., S. 33. 
Die Gruppeninterviews werden unter Zuhilfenahme eines Leitfadens geführt. Ein Leitfaden dient hierbei der Fokussierung auf die Fragestellung und soll sicherstellen, dass alle dafür relevanten Themenbereiche zur Sprache kommen. Ein Nachteil eines strukturierten Interviews mit Leitfaden ist, dass es die Teilnehmenden in ihren Antworten einschränkt. Infolge der vergleichsweise starken Steuerung des Gesprächs durch die Forscherin bleiben Themen, die nicht vorhersehbar waren und deswegen keinen Eingang in den Leitfaden fanden, eventuell außen vor. Dennoch soll die Befragung hier nicht in Form eines narrativen Interviews erfolgen, weil der Fokus der Forschung sehr klar ist. Anders als beispielsweise bei einer Biographiearbeit bildet die Bürgerwehr einen sehr klaren Fokus, der die einzelnen Mitglieder der Bürgerwehr vereint.

Eine Analyse schriftlicher Quellen ist für eine Studie über Bürgerwehren nur sehr eingeschränkt möglich. Wie bereits erwähnt, produzieren Bürgerwehren kaum eigene Texte, und eine Analyse von Medienberichten über Bürgerwehren würde das Phänomen stets durch die Brille der Journalist*innen betrachten. Einzig Sozial-Media-Posts der Mitglieder der Bürgerwehr, beispielsweise auf Facebook oder Twitter, liefern eine schriftliche Quelle, die für die Studie relevant sein könnte. Nicht alle Bürgerwehren sind jedoch gleichermaßen in den Sozialen Medien präsent. Insbesondere bei den Typ IIIBürgerwehren stellt eine Analyse von Social-Media-Posts jedoch eine fruchtbare Ergänzung dar, weil sich hier Haltungen zeigen, die in der Anwesenheit der Forscherin aus Gründen der politischen Opportunität zurückgehalten wurden.

Das folgende Kapitel diskutiert nun zunächst die ethnografische Herangehensweise für politikwissenschaftliche Forschung, um daran anschlieBend Feldforschung als methodischen Zuschnitt der Untersuchung darzulegen. Das zu analysierende Datenmaterial wird über eine teilnehmende Beobachtung sowie durch Leitfaden-strukturierte Interviews mit den Mitgliedern der Bürgerwehren im Feld generiert. Dabei bedient sich die Untersuchung dem Ansatz der »dichten Beschreibung « von Clifford Geertz. Für die Analyse wird das Datenmaterial codiert und die Codes anschließend zu Codeklassen zusammengefasst. 


\subsection{Dissolving the a priori breach between theory and method: Zum Forschungsansatz der Ethnographie}

Ethnografische Forschung sucht den direkten Zugang, will Einblicke in das spezifisch subjektive Wissen der Subjekte erlangen. Dabei erfolgt die analytische Beschreibung der sozialen Situationen und der in ihnen handelnden Subjekte mit dem Ziel, diese so zu repräsentierten, dass sich Leser*innen ein Bild von dem beschriebenen Phänomen machen können. ${ }^{3}$

Für Bürgerwehren als klandestines Phänomen ist diese Beschreibung der handelnden Akteure eine Möglichkeit, einen Zugang zu den inneren Logiken zu bekommen, denen das Handeln und Denken der Mitglieder folgt. Ethnografie geht davon aus, dass es eine Ordnung gibt, die das Soziale organisiert wir müssen nur Wege finden, sie zu beschreiben. Damit bewegt sich der Ansatz in einem Spannungsfeld zwischen Naturalismus und Konstruktivismus. Einerseits nimmt er qua Methode an, dass es real existierende »Felder« gibt, die eine Ordnung aufweisen. Andererseits hat die Disziplin Ethnologie im Laufe ihrer Geschichte ein hohes Maß an Sensibilität entwickelt, inwieweit sie selber zur Konstruktion der untersuchten Phänomene, Orte und Gesellschaften beiträgt. ${ }^{4}$

Als nicht-standardisierter Forschungsansatz beruht die Ethnografie auf einem starken Empiriebegriff. Die Vorstellung eines »natürlichen Laboratoriums« wird ebenso verworfen wie die Idee neutraler Rohdaten, die »da draußen « zu finden sind. Es wird vielmehr davon ausgegangen, dass es nicht möglich ist, die abhängigen von den unabhängigen Variablen abzusondern. ${ }^{5}$ Ethnografie »dissolves the a priori breach between theory and method «. ${ }^{6}$ Theorie und Methode sind nicht voneinander zu trennen und betreiben ein unaufhörliches Wechselspiel: Aus der methodischen Herangehensweise lässt sich Theorie generieren, die wiederum den methodischen Zugang verändert.

Mehr als eine Methode ist Ethnografie eine Haltung und eine Forschungsstrategie. Sie will sich dem Forschungsgegenstand empirisch nähern und ihn

3 Vgl. Breidenstein, Georg et al. (2013): Ethnographie. Eine Praxis der Feldforschung. Konstanz: UVK. S. 7.

Vgl. ebd., S. 10.

Vgl. Geertz, Clifford (1987): Dichte Beschreibung. Frankfurt a.M.: Suhrkamp. S. 32f.

Comaroff, Jean/Comaroff, John (2003): Ethnography on an Awkward Scale: Postcolonial Anthropology and the Violence of Abstraction. In: Ethnography 2003, 4. S. 172. 
in seiner Vielschichtigkeit und Widersprüchlichkeit abbilden. ${ }^{7}$ Diese starke Stellung der Empirie in der ethnografischen Forschung bedeutet keineswegs eine bloße Reproduktion der Sichtweisen der untersuchten Subjekte. Ihr Verhalten und ihre Perspektive dienen der Beschreibung einer das Soziale regulierenden Struktur und bedürfen daher der interpretativen Analyse der Forscherin. Dieser Aspekt ist insbesondere für die Untersuchung von Bürgerwehren zentral. So finden sich unter den Mitgliedern von Bürgerwehren nicht selten Menschen mit einem rechtsextremen Weltbild. Diese politischen Positionen sind für das Verständnis von Bürgerwehren wichtig, sie dürfen jedoch nicht einfach nur repräsentiert werden. Vielmehr bedarf es hier des analytischen Blicks der Wissenschaftlerin, um eine Legitimierung oder Normalisierung dieser Positionen zu vermeiden (vgl. hierzu Kapitel 4.1.2.2).

Die Haltung hinter dem ethnografischen Forschungsansatz hat sich historisch jedoch sehr verändert. Als die Forschungsrichtung im Zuge der Aufklärung in Europa Ende des 18. Jahrhunderts aufkam, beschäftigte sie sich unter dem Namen »Völkerkunde« beziehungsweise »Volkskunde« mit der Beschreibung von Gesellschaften außerhalb Europas. ${ }^{8}$

Im 19. und 20. Jahrhundert war die Volkskunde sowohl personell und organisatorisch als auch ideell und politisch eng mit dem Kolonialismus verwoben. ${ }^{9}$ Mit ihrer exotisierenden Beschreibung "primitiver Kulturen « ließ sie sich vor den Wagen der politischen Legitimation kolonialer Herrschaft spannen. Nicht selten waren die Wissenschaftler selber Teil des kolonialen Apparates. Auch noch in der zweiten Hälfte des 20. Jahrhunderts, nachdem die meisten Kolonien ihre Unabhängigkeit erlangt hatten, wohnten der Erforschung lokaler Gesellschaften in Ländern des Südens rassistische Projektionen inne.

Mit seinem 1978 erschienenen Werk Orientalism kritisierte der USamerikanische Literaturtheoretiker Edward Said die Konstruktion des "Orients« durch die westliche Welt. Er beschrieb das im Zusammenhang von Wissen(schaft) und Macht vollzogene »Othering « als einen Prozess, in dem »das Fremde« konstruiert wird, um das Eigene zu definieren.

7 Vgl. Breidenstein, Georg et al. (2013): Ethnographie. Eine Praxis der Feldforschung. Konstanz: UVK. S. $8 \mathrm{f}$.

8 Vgl. Kaschuba, Wolfgang (2006): Einführung in die Europäische Ethnologie. München: Beck. S. 21.

9 Vgl. Breidenstein, Ceorg et al. (2013): Ethnographie. Eine Praxis der Feldforschung. Konstanz: UVK. S. 14. 
»The Orient has helped to define Europe (or the West) as its contrasting image, idea, personality, experience. ${ }^{10}$

Die Ethnologie traf diese Kritik besonders hart. Waren doch in der Beschreibung postkolonialer Gesellschaften die kolonial geprägten rassistischen Denkweisen besonders offensichtlich. Die vielen Ethnologen in den ehemaligen Kolonien bildeten einen Definitionsapparat, der kulturelle Merkmale der lokalen Gesellschaften essentialistisch beschrieb und festschrieb. Dieser Prozess geschah dabei keineswegs immer unterbewusst. Bronislaw Malinowski, einer der Gründungsväter der Sozialanthropologie, notierte 1917 in Neuguinea:

»Besitzgefühl: Ich bin es, der sie beschreiben oder erschaffen wird. ${ }^{11}$

Im Anschluss an Saids Kritik entwickelte sich auch in der Ethnologie eine selbstkritische Debatte über die Rolle der Disziplin in der Fortschreibung rassistischer Denkmuster.

Die sogenannte Writing Culture-Debatte der 1980er Jahre legte zwei grundlegende Problematiken ethnografischen Forschens frei. Einerseits wurde die ethnografische Repräsentation diskutiert: ${ }^{12}$ Was kann überhaupt abgebildet werden? Lässt sich aus beobachtetem Verhalten überhaupt ein Regelsystem ableiten, das Kultur genannt werden kann? Die zweite Ebene befasste sich mit dem Akt des Beschreibens selbst. So wurde der unvermeidbare hierarchische Bestandteil ethnografischer Forschung aufgedeckt: Ein "Sprechen von« ist immer auch ein »Sprechen für ${ }^{13}$

Die klassische Ethnologie verstand sich als positivistische Wissenschaft. Sich an den Naturwissenschaften orientierend, wurde das subjektive Moment der Beschreibung einer (fremden) Kultur ausgeblendet. Mit der Writing

10 Said, Edward (1977): Orientalism. London: Penguin. S. 1f.

11 Malinowski, Bronislaw (1917): Ein Tagebuch im strikten Sinne des Wortes. Neuguinea 1914-1918. Frankfurt a.M. (1985), S. 127. Zitiert nach: Gottowik, Volker: Zwischen dichter und dünner Beschreibung: Clifford Geertz' Beitrag zur Writing Culture-Debatte. In: Därmann, Iris/Jamme, Christoph (Hg.): Kulturwissenschaften. Konzepte, Theorien, Autoren. München: Verlag? S. 122.

12 Vgl. Meyer, Christian (2009): Ereignisethnographie und Methodologischer Situationalismus. Auswege aus der Krise der ethnographischen Repräsentation. In: Berger, Peter et al.: Feldforschung. Ethnologische Zugänge zu sozialen Wirklichkeiten. Berlin: Weißenseeverlag. S. 403.

13 Vgl. Breidenstein, Georg et al. (2013): Ethnographie. Eine Praxis der Feldforschung. Konstanz: UVK. S. 19. 
Culture-Debatte wurde die literarische Rolle der Ethnograf*innen in den Fokus gerückt: In der Beschreibung von Kultur geschieht gleichzeitig eine Festschreibung von Kultur.

»Ethnographic truths are thus inherently partial - committed and incomplete ${ }^{14}$, schreibt James Clifford in der Einleitung des von ihm herausgegebenen Sammelbands »Writing Culture«, der zentrale Stimmen der Debatte zusammenbringt.

»The writing and reading of ethnography are overdetermined by forces ultimately beyond the control of either an author or an interpretive community. These contingencies - of language, rhetoric, power, and history - must now be openly confronted in the process of writing. ${ }^{15}$

Den Anstoß für die Writing Culture-Debatte liefert Clifford Geertz. In einer Fußnote seines Essays »Dichte Beschreibung. Bemerkungen zu einer deutenden Theorie von Kultur ${ }^{16}$ schreibt Geertz:

»Eine Reflexion über die Darstellungsweisen (von Experimenten [...] nicht zu sprechen) hat in der Ethnologie sehr gefehlt. «" ${ }^{17}$

Diese Fußnote sollte die Ethnologie revolutionieren. ${ }^{18}$ Sie lieferte den Anstoß für eine Konferenz in Santa Fe, New Mexico, die sich mit dem Vorwurf auseinandersetzte, die Disziplin reflektiere ihre Herangehensweise bei der Beschreibung von Kultur nicht. Die Beiträge der Konferenz bilden den Sammelband »Writing Culture ${ }^{19}$

Das Projekt der Selbstreflexion beinhaltete auch ein Bewusstwerden des »Othering", das in ethnografischen Studien betrieben wurde, also der kli-

Clifford, James (Hg.) (1986): Writing Culture. The Poetics and Politics of Ethnography. Berkeley: University of California Press. S. 6.

15 Ebd., S. 25.

16 Vgl. Geertz, Clifford (1973/1987): Dichte Beschreibung. Bemerkungen zu einer deutenden Theorie von Kultur. In: Geertz, Clifford (1987): Dichte Beschreibung. Frankfurt a.M.: Suhrkamp. S. 9.

17 Vgl. Ceertz, Clifford (1987): Dichte Beschreibung. Frankfurt a.M.: Suhrkamp. S. 28f. Fußnote 3 .

18 Vgl. Gottowik, Volker: Zwischen dichter und dünner Beschreibung: Clifford Geertz' Beitrag zurWriting Culture-Debatte. In: Därmann, Iris/Jamme, Christoph (Hg.): Kulturwissenschaften. Konzepte, Theorien, Autoren. München. S. 119ff.

19 Clifford, James (Hg.) (1986): Writing Culture. The Poetics and Politics of Ethnography. Berkeley: University of California Press. 
scheehaften Projektion all jener Eigenschaften, die nicht im eigenen Selbstverständnis verortet werden sollten, auf die fremden Gesellschaften. »The deconstruction of otherness is the price of truth, ${ }^{20}$ schreibt Clifford Geertz. Diese Dekonstruktion führt jedoch nicht zu einer objektiven Stimme, in der es der Ethnografin gelingt, jegliche Projektionen und Essentialisierungen aus der Beschreibung zu verbannen. Vielmehr wird Subjektivität als zentraler Bestandteil ethnografischer Forschung anerkannt:

»The writer's >voice< pervades and situates the analysis, and objective, distancing rhetoric is renounced. $\ll^{21}$

Damit wurde es zur zentralen Aufgabe der Ethnologie, Strategien für einen reflektierten Umgang mit dieser Subjektivität zu entwickeln.

Die Debatte fiel in die Zeit des >cultural turn in der Ethnologie und trug $\mathrm{zu}$ einer radikalen Neuorientierung bei. Und sie machte den Weg frei für zahlreiche neue Ansätze. ${ }^{22}$ So lenkten Hausschild/Nixdorf mit ihrem 1982 erschienenen Sammelband »Europäische Ethnologie« den Fokus bewusst weg vom Fremden in Ländern des Südens auf die ethnografische Betrachtung europäischer Gesellschaften. ${ }^{23}$ Die Multi-Sited Ethnography ${ }^{24}$ entwickelte eine Möglichkeit, translokale Phänomene wie Grenzkriminaliät und Migration zu analysieren. Und D. Soyini Madison beschrieb seinen Ansatz der kritischen Ethnologie als eine Praxis der Kritischen Theorie. ${ }^{25}$

In der jüngeren Forschung findet Ethnografie als Methode auch Eingang in andere Sozialwissenschaften. In den Politikwissenschaft ist die Herangehensweise jedoch noch vergleichsweise neu. Da ethnografische Forschung

20 Geertz, Clifford (2000): Anti-anti-relativism. In: Ders: Available Light. Anthropological Reflections on Philosophical Topics. New Jersey: Princeton University Press. S. 63.

21 Clifford, James (Hg.) (1986): Writing Culture. The Poetics and Politics of Ethnography. Berkeley: University of California Press. S. 12.

22 Vgl. Breidenstein, Georg et al. (2013): Ethnographie. Eine Praxis der Feldforschung. Konstanz: UVK.S. 2off.

23 Vgl. Hauschild, Thomas/Nixdorf, Heide (Hg.) (1982): Europäische Ethnologie. Theorieund Methodendiskussion aus ethnologischer und volkskundlicher Sicht. Berlin: Dietrich Reimer Verlag.

24 Vgl. Marcus, George E.: Ethnography in/of the world system: The Emergence of MultiSited Ethnography. In: Annual Review of Anthropology. Vol. 24 (1995). S. 95-117.

25 Vgl. Madison, D. Soyini (2004): Critical Ethnography. Method, Ethics, and Performance. Los Angeles: Sage. S. 15. 
über das Lokale hinaus angewandt werden kann und die daraus gewonnenen Beobachtungen zudem als in Makro-Strukturen eingebettet analysiert werden können, erweist sich ethnografische Forschung auch in den internationalen Beziehungen als produktiv - beispielsweise bei der Betrachtung transnationaler Mobilität. ${ }^{26}$

Der >cultural turn in der Ethnologie lässt die Ethnografie für die Untersuchung von Bürgerwehren überhaupt erst in Betracht kommen. Denn bei der Beschreibung der handelnden Subjekte als Bürgerwehren muss darauf geachtet werden, die Gruppen nicht vorschnell aufgrund von Vorurteilen gesellschaftlich etablierten Kategorien zuzuordnen. Vielmehr muss sich eine Analyse eng am Datenmaterial orientieren und nur hieraus Schlüsse generieren (vgl. hierzu Kapitel 4.2.3).

Ebenso wichtig für eine Anwendung in der Untersuchung von Bürgerwehren ist, dass ethnologische Forschung mit einem Politikbegriff operiert, der das Politische in den konkreten Lebenszusammenhängen verortet. ${ }^{27}$ Verbreitet ist hier der Ansatz der lebensweltlichen Ethnologie. ${ }^{28}$ Dieser ist jedoch sehr unpolitisch, weil die Analyse weitgehend auf der Mikro- und Mesoebene verbleibt. Für den hier angelegten Analyserahmen ist jedoch eine Berücksichtigung von Phänomenen der Makroebene unerlässlich.

Diese Studie wählt daher den Ansatz der »dichten Beschreibung « von Clifford Geertz. Dieser vermag die Mikro- mit der Makroebene zu verknüpfen (vgl. Kapitel 4.2.1). Die Untersuchung von Bürgerwehren in Deutschland ist eine ethnografische Studie innerhalb der eigenen Gesellschaft der Forscherin. Diese Herangehensweise soll anstatt von Ethnie und Kultur andere Kategorien wie Geschlecht, Schicht, politische Werte als Beschreibungsdimensionen individueller Identität zur Erklärung des Phänomens heranziehen ${ }^{29}$ und somit dem historisch gewachsenen Fetisch der Ethnie in der Ethnologie entgegenwirken - denn der Kulturbegriff neigt bis heute dazu, soziale Phänomene

Vgl. Sabaratnam, Meera (2017): Symposium: Walking with Migrants. Ethnography as Method in International Relations, Harvard Dataverse, V1. Online unter: https://doi.or g/10.7910/DVN/WUK8AN.

Vgl. Horak, Roman/Spitaler, Georg (2002): »Das Politische« im Feld. Über Ethnographie und die Möglichkeiten politikwissenschaftlicher Kulturstudien. In: Österreichische Zeitschrift für Politikwissenschaft, 31 (2002) 2. S. 201.

28 Vgl. bspw. Honer, Anne (2011): Kleine Leiblichkeiten. Erkundungen in Lebenswelten. Wiesbaden: VS Verlag. S. 60.

29 Vgl. Kaschuba, Wolfgang (2006): Einführung in die Europäische Ethnologie. München: Beck. S. 147. 
zu ethnisieren. ${ }^{30}$ Kultur soll als Struktur begriffen werden, in der Handlungen Sinn ergeben (vgl. Kapitel 4.2.1) - hier spielen andere Dinge eine viel wichtigere Rolle als die Ethnie der untersuchten Subjekte.

\subsubsection{Methodischer Zuschnitt Feldforschung: Ein Zugang zur Sozialität der Situation}

Der Zugang zum Feld zeichnet sich durch eine Unvoreingenommenheit aus. Die Forscherin lässt sich auf die Logik des Feldes ein und sucht das Wissen der Teilnehmer*innen der Situation. Dennoch geht man keineswegs unvorbereitet ins Feld. ${ }^{31}$ Der Feldzuschnitt beinhaltet eine Festlegung auf ein Thema und einen Analyserahmen (vgl. Kapitel 2) sowie die Wahl des räumlichen, zeitlichen und sozialen Untersuchungsausschnitts (vgl. Kapitel 3 und 5). In diesem Zuge werden außerdem gesellschaftliche und politische Rahmendaten recherchiert (vgl. Kapitel 6.1, 7.1 und 8.1). Auf dieser Grundlage können überhaupt erst Arbeitshypothesen entstehen, die vor Ort bearbeitet werden können. Bei der konkreten Datenerhebung im Feld herrscht ein »feldspezifischer Opportunismus «. ${ }^{32}$ Je nach Beschaffenheit des Feldes muss entschieden werden, welche Methoden überhaupt möglich sind und welche sich besonders für die Untersuchung der Hypothesen eignen (vgl. Kapitel 4.1.1.1 und 4.1.1.2).

Eine Gefahr bei der Feldforschung soll an dieser Stelle nicht unerwähnt bleiben. Feldforschung ist auch Ausdruck einer Faszination des Fremden. Sie befriedigt die Anziehungskraft, die Marginalisierte auf westliche Forscher*innen ausüben. Dabei erscheint »das Andere« erst in Abgrenzung zur eigenen Lebenswelt spannend. ${ }^{33}$ Die Kritik richtet sich insbesondere gegen eine unreflektierte Exotisierung von Gesellschaften in Ländern des Südens. Mit dem »cultural turn« ist diese chauvinistische Forschungshaltung zwar

30 Vgl. Breidenstein, Georg et al. (2013): Ethnographie. Eine Praxis der Feldforschung. Konstanz: UVK. S. 31.

31 Zur Kritik am Begriff des »Feldes« siehe: Gupta, Akhil/Ferguson, James (Editor) (Hg.) (1997): Anthropological Locations Boundaries and Grounds of a Field Science. Berkeley: University of California Press.

32 Vgl. Breidenstein, Georg et al. (2013): Ethnographie. Eine Praxis der Feldforschung. Konstanz: UVK. S. 34.

33 Vgl. Greverus, Ina-Maria: Die Sehnsucht des Ethnologen nach dem Feld. In: Hauschild, Thomas/Nixdorf, Heide (Hg.) (1982): Europäische Ethnologie. Theorie- und Methodendiskussion aus ethnologischer und volkskundlicher Sicht. Berlin: Dietrich Reimer Verlag. S. $213 f$. 
einem Selbstreflexionsprozess unterworfen, der sich in die ethnografische Forschung eingliedert, dennoch sind ethnografische Studien auch heute nicht frei von rassistischen Projektionen. Aber auch bei Forschungsvorhaben innerhalb der eigenen Gesellschaft gilt es, die Motivationen der Forscherin bei der Wahl der Feldforschung als methodische Herangehensweise kritisch zu prüfen (vgl. Kapitel 4.1.2).

Für die Untersuchung der Bürgerwehren wird eine teilnehmende Beobachtung gewählt. Das Auftreten der Mitglieder der Bürgerwehr im Öffentlichen Raum konstituiert die Bürgerwehr erst als solche. Die Patrouille bildet daher die Situation, die es zu untersuchen gilt, um dem Phänomen Bürgerwehr gerecht zu werden. Zudem werden Leitfaden-strukturierte Feldinterviews mit den Mitgliedern der Bürgerwehr geführt. Im Folgenden wird nun die für die Untersuchung gewählte Methodik diskutiert.

\subsubsection{Das Phänomen am eigenen Leib erfahren: Teilnehmende Beobachtung als Forschungsmethodik}

Teilnehmende Beobachtung zeichnet sich durch eine sinnliche Unmittelbarkeit aus. Die Forscherin sucht die Situation, die es zu untersuchen gilt, physisch auf und begibt sich in sie hinein. Die Bürgerwehren werden auf ihrer Patrouille begleitet. In dem Moment, da sie sich durch ihr Präsent-werden im öffentlichen Raum als Bürgerwehr konstituieren, verändern sie ihr Verhalten, sie werden vom Bürger, der abends durch die Straßen spaziert, zum Mitglied der Bürgerwehr und als solches zu einem Sicherheitsakteur. Ihr Verhalten verändert sich mit der neuen Rolle, und auch Verhaltensweisen, die sie in ihrer Rolle als normaler Bürger ebenso an den Tag gelegt hätten, bekommen im Kontext der Patrouille eine andere Bedeutung.

Diese Veränderungen gilt es zu analysieren, und dafür ist es unabdingbar, in der Situation anwesend zu sein. Jedoch verändert die Anwesenheit notwendigerweise die Situation und das Verhalten der Subjekte. Um diese Veränderung zu minimieren, ist der Versuch, möglichst wenig aufzufallen und sich im Hintergrund zu halten, nicht zielführend. Dies verstärkt nur die Unsicherheit der Mitglieder der Bürgerwehr in der Situation. Vielmehr gilt es, durch aktive Teilnahme eine Normalität herzustellen, die es den Akteuren ermöglicht, sich weitestgehend authentisch zu verhalten.

Auf Seiten der Ethnografin ist jedoch ein völliges Aufgehen in der Situation nicht hilfreich. Es darf zu keiner bedingungslosen Identifizierung mit den handelnden Individuen kommen. Die Forscherin bewegt sich im Span- 
nungsfeld zwischen Teilnahme und Distanz, zwischen Präsent-sein und Repräsentieren. ${ }^{34}$ Dieses Spannungsfeld wird insbesondere deutlich, wenn es zu Situationen kommt, in denen Handeln moralisch geboten ist. Wenn die Bürgerwehr beispielsweise Gewalt anwendet, liegt es an der Forscherin, ihre Rolle zu verlassen und einzugreifen, auch wenn das zu einer Verfälschung der $\mathrm{zu}$ beobachtenden Situation führt.

Teilnehmende Beobachtung scheint ein ambiguer Begriff zu sein, stehen doch die Handlungen »Teilnehmen « und "Beobachten« in einem gewissen Widerspruch zueinander. ${ }^{35}$ Wer teilnimmt, ist beschäftigt, kann weniger genau beobachten, was um ihn oder sie herum geschieht. Gleichzeitig erschließen sich soziale Phänomene aus der Perspektive eines Teilnehmenden auf eine andere Weise als aus der Beobachtungsperspektive. Dieses eigene Involviertsein, »es am eigenen Leib erfahren«, um dieses Erfahrungswissen geht es bei der teilnehmenden Beobachtung.

So ist die Ethnografin gleichzeitig Teilnehmerin und Beobachterin, sie springt zwischen den beiden Positionen hin und her und vereint sie in dem Moment, wo sie ihre eigenen Erfahrungen in der Situation beobachtet. Die Forschungsfragen laufen dabei immer im Hinterkopf mit, das eigene Wertesystem analysiert das Geschehen. Nicht zuletzt besteht zwischen der Betrachterin und den Betrachteten ein Machtverhältnis, das ein völliges Auflösen in der Situation verhindert. Die Forscherin ist diejenige, die die anderen beschreibt, ihr Handeln interpretiert.

Bei der Vereinbarkeit zwischen Teilnahme und Beobachtung kann die Rolle, die der Forscherin im Geschehen spielt, variiert werden. Bei einer starken Teilnahme gerät die Gesamtperspektive aus dem Blickfeld und es ist schwieriger, das Erlebte (schriftlich oder auditiv/visuell) aufzuzeichnen. Das ist bei einer schwachen Teilnahme viel besser möglich. ${ }^{36}$ Es ist also ratsam, während der teilnehmenden Beobachtung die soziale Position zu variieren. ${ }^{37}$ Darüber hinaus ist es wichtig, offen zu sein für das scheinbar Abseitige und Unwich-

\footnotetext{
34 Vgl. Breidenstein, Georg et al. (2013): Ethnographie. Eine Praxis der Feldforschung. Konstanz: UVK. S. 7

35 Vgl. ebd., S. 66.

36 Vgl. ebd., S. 67.

37 Vgl. ebd., S. 79.
} 
tige. Die Forscherin muss Situationen quer zu den vordergründigen Sinnzusammenhängen der Teilnehmenden lesen können. ${ }^{38}$

Mitunter verbringt eine Ethnografin lange Zeit im Feld, teilweise wird es über Jahre immer wieder aufgesucht. Ein derart tiefes Einsteigen in die sozialen Gefüge kann im Fall der Bürgerwehren kaum erlangt werden, weil sich die Bürgerwehren in unterschiedlichen zeitlichen Abständen treffen und nur für ein oder zwei Stunden für die Patrouille zusammenkommen und danach wieder ihrer Wege gehen und in andere soziale Rollen schlüpfen.

Auch eine Akkumulation von Felderfahrung ist im Falle der Bürgerwehren häufig nicht möglich. Das Phänomen tritt äußerst sprunghaft auf und verschwindet mitunter innerhalb kurzer Zeit wieder, wenn die Teilnehmenden das Interesse an der Bürgerwehr verlieren (vgl. Kapitel 5).

Für die Untersuchung der Bürgerwehren erfolgt die teilnehmende Beobachtung auf der Patrouille und verläuft entlang der Route, die die Bürgerwehr wählt. Sie endet, wenn die Mitglieder auseinander gehen. Das heißt, wenn beispielsweise anschließend als Gruppe noch ein Bier getrunken wird, so ist auch das noch Teil des untersuchten Zeitrahmens. Denn auch hier tritt die Bürgerwehr noch als solche auf. Auch die Kneipe ist ein Raum, in dem die Mitglieder der Bürgerwehr als solche agieren würden, sollte sich eine Situation ergeben, in der sie sich zum Handeln veranlasst sehen. Zudem ist in diesem Rahmen unter Umständen ein lockerer Austausch über die Patrouille zu beobachten, oder die Teilnehmenden unterhalten sich über ihre Motivationen, der Bürgerwehr beigetreten zu sein.

Im Vorfeld der Untersuchung stellt sich die Frage des Feldzugangs. Nachdem ein geeigneter sozialer, örtlicher und zeitlicher Rahmen gewählt wurde, müssen die agierenden Personen des Felds kontaktiert werden. Ein Kontakt lässt sich über die Präsenz der Bürgerwehren in den sozialen Medien herstellen. Teilweise werden Mitglieder der Bürgerwehr in Zeitungsartikeln zitiert und dabei mit vollem Namen erwähnt.

Geeignete Bürgerwehren zu finden, die bereit sind, sich auf ihren $\mathrm{Pa}$ trouillen begleiten zu lassen, stellt jedoch insbesondere bei Bürgerwehren, die in rechte Strukturen eigebunden sind, eine gewisse Schwierigkeit dar. Denn in diesem Milieu herrscht eine Skepsis gegenüber offiziellen Institutionen, zu denen auch die Universität gehört. Andererseits ist die Begleitung auch Ausdruck einer Wertschätzung des Engagements der Bürgerwehr. An dieses

38 Vgl. Breidenstein, Georg et al. (2013): Ethnographie. Eine Praxis der Feldforschung. Konstanz: UVK. S. 71. 
Bedürfnis nach Anerkennung und Aufmerksamkeit soll bei der Kontaktaufnahme angeknüpft werden. Der Feldzugang wird im Rahmen der Einzelfallstudien in Kapitel 6.1.1, 7.1.1 sowie 8.1.1 genauer erläutert.

Nach der teilnehmenden Beobachtung beginnt der Prozess, in dem die Bedeutung des Schreibens in der Ethnografie deutlich wird. Die Erfahrungen der Forscherin bei der teilnehmenden Beobachtung werden verschriftlicht. Es müssen Worte für die sinnlichen Erfahrungen gefunden werden (vgl. Kapitel 4.2.1). Anschließend erfolgt ein Distanzierungsprozess, in dem sich die Forscherin von der unmittelbaren Erfahrung löst und eine analytische Perspektive einnimmt. Die dichten Beschreibungen der Situationen, bei denen die Bürgerwehren begleitet wurden, werden dann systematisch analysiert (vgl. Kapitel 4.2.1).

\subsubsection{Von der Handlungsebene zur Textebene: Leitfaden-strukturierte (Gruppen-)Interviews im Feld}

Die teilnehmende Beobachtung wird ergänzt durch Leitfaden-gestützte Interviews im Feld. Die Feldsituation bleibt bestehen, doch existiert auch eine Interviewsituation. Die Forscherin stellt Fragen und nimmt das Interview mit einem Aufnahmegerät auf. Auch, wenn die teilnehmende Beobachtung im gewohnten Umfeld für die Mitglieder der Bürgerwehr stattfindet, ist die Interviewsituation künstlich geschaffen. Die Bürgerwehr erzählt allein auf Bitten der Forscherin und bekommt durch die Fragen Impulse. Mit den Fragen werden die Themen gesteuert, über die die Mitglieder der Bürgerwehr diskutieren. Dieser künstlich geschaffene Rahmen beeinflusst, auf welche Art und Weise Aussagen getätigt werden.

Jede soziale Situation, in der gesprochen wird, lässt sich als ein spezielles »speech event« kategorisieren. Wir unterscheiden zum Beispiel ganz selbstverständlich zwischen einem Einstellungsgespräch und einer Unterhaltung unter Freund"innen. Jede dieser Sprechsituationen folgt ihren eigenen Regeln. So auch das Interview. ${ }^{39}$ Im Gegensatz zu einer freundlichen Unterhaltung unter Bekannten steuert die Interviewerin den Gesprächsfluss. Sie steuert sehr direkt durch ihre Fragen, aber auch durch Augenkontakt, aufmunterndes Nicken oder den scheinbar desinteressierten Blick auf die eigenen Notizen. 
Die Interviewerin ist in der Interviewsituation sehr spezifisch Adressatin der Aussagen. Ihre konkrete Person wirkt dabei auf die Teilnehmenden (vgl. Kapitel 4.1.4), aber auch die Interviewsituation als solche beeinflusst, wie geantwortet wird. Das Setting einer wissenschaftlichen Studie beeinflusst die Wortwahl der Interviewpartner*innen und lässt sie Dinge zurückhalten, die sie in einer lockeren Unterhaltung erzählen würden - entweder weil sie diese für wissenschaftlich uninteressant oder für zu privat halten. Hier ist es Aufgabe der Interviewerin, mit geschickt formulierten Fragen dieses Wissen hervorzubringen und eine Atmosphäre zu schaffen, in der sich die Teilnehmenden sicher fühlen.

Während sich die Beobachtung insbesondere dazu eignet, Handlungsschemata $\mathrm{zu}$ interpretieren und routinierte Körperpraktiken $\mathrm{zu}$ beschreiben, lassen sich durch Interviews vor allem subjektive Wissensbestände und Deutungen für die beobachteten Handlungen rekonstruieren. ${ }^{40}$ Die FrageAntwort-Situation ist eine künstlich geschaffene, es geht hier nicht darum, die Mitglieder der Bürgerwehr möglichst authentisch zu erleben. Diese Aufgabe erfüllt die teilnehmende Beobachtung, durch sie kommt man ganz nah heran. Die Interviews im Feld erfassen die Gruppeninteraktionsprozesse, und die Antworten der Teilnehmenden geben Aufschluss über ihre Selbstkonzepte und ermöglichen Zugang zu ihrem Erfahrungsschatz.

So wird beispielsweise nach der Gründungsphase der Bürgerwehr gefragt. Es wird deutlich, welche Narrative die Mitglieder von sich und ihrem Handeln als Bürgerwehr haben, wie sie den Ort wahrnehmen, an dem sie leben, was Sicherheit für sie bedeutet. Die politischen Einstellungen der Bürgerwehr werden erfasst, indem danach gefragt wird, was die Mitglieder mit Polizei, Kriminalität und Strafe verbinden.

Der Vorteil an Interviews im Feld gegenüber einer künstlich geschaffenen Interviewsituation, die losgelöst von der teilnehmenden Beobachtung ist, besteht darin, dass diese informell und locker wirkenden Gespräche einen emotionalen Zugang zu den Teilnehmenden ermöglichen. Die Gespräche finden in der Situation statt, und die Personen können sich direkt auf ihr Handeln beziehen. Der direkte Zugang zur körperlichen Erfahrung ist also noch gegeben, während er in einem späteren Interview von den Personen erneut gedanklich abgerufen werden muss. Im Feld-Setting ist es daher besonders günstig, unbewusstes Wissen zu erfragen. Eine spontane Antwort bringt

40 Vgl. Honer, Anne (2011): Kleine Leiblichkeiten. Erkundungen in Lebenswelten. Wiesbaden: VS Verlag.S. 60. 
diesen Erfahrungsschatz eher hervor als eine überlegte und wohlformulierte Antwort in einer Interviewsituation. ${ }^{41}$

Auch Interviews im Feld folgen einem strukturierten Leitfaden (siehe Kapitel 4.2.2) - er dient jedoch lediglich der Fokussierung auf die Fragestellung der Studie. Die Diskussionen in der Gruppe entwickeln sich eigenständig und weichen mitunter vom Leitfaden ab. Argumentationen werden bewusst laufen gelassen, aber der Leitfaden stellt sicher, dass die Einstellungen der Mitglieder zum Themenbereich »Sicherheit« Eingang in die Studie finden.

Gleichzeitig ermöglicht die Feldsituation ein lockeres Plaudern, das den Interviewten nicht das Gefühl gibt, ausgefragt zu werden..$^{42}$ In der Dynamik der Situation entstehen möglicherweise Aussagen über die politische Ausrichtung der Mitglieder, die in einer Interviewsituation dem Reflexionsprozess vor der Antwort zum Opfer fallen würden.

Insbesondere die Bürgerwehren zweiten und dritten Typs gehen mit ihrem Engagement mitunter nicht offen um. Bürgerwehren müssen mit Gegenmaßnahmen seitens der Polizei oder negativer Berichterstattung in der Presse rechnen. Es gibt daher Mitglieder, die nicht mit der Bürgerwehr assoziiert werden wollen, weil sie Nachteile - zum Beispiel im Job - befürchten.

Weil nicht alle Mitglieder bereit sind, sich dem Interview anzuschließen und ihre Aussagen aufzeichnen zu lassen, muss auf Interviews mit für die Bürgerwehr relevante Einzelpersonen zurückgegriffen werden. Dies sind entweder die Gründer der Bürgerwehr oder besonders engagierte Mitglieder, die eine Organisatorenrolle einnehmen und sich berufen fühlen, für die Gruppe zu sprechen. Die jeweils spezifischen Bedingungen des Zugangs zum Feld werden im Rahmen der Einzelfallstudien noch genauer erörtert (vgl. Kapitel 6.1.1, 7.1.1 und 8.1.1).

\subsubsection{Forschungsethik: Zur Vereinbarkeit von Forschungsinteressen und ethischen Standards zum Schutz der involvierten Individuen}

Jede Forschung bewegt sich im Spannungsfeld zwischen Forschungsinteressen und der Einhaltung ethischer Standards. In der empirischen Sozialforschung geht es dabei insbesondere um den Schutz der Individuen, die mit

\footnotetext{
41 Vgl. Breidenstein, Georg et al. (2013): Ethnographie. Eine Praxis der Feldforschung. Konstanz: UVK.S. $81 f$.

42 Vgl. ebd., S. 80.
} 
ihren Aussagen oder Handlungen zum Datenmaterial beitragen. Das Einholen einer informierten Zustimmung der Teilnehmenden ist dabei ein zentraler ethischer Grundsatz.

Bürgerwehren als Untersuchungsgegenstand sind schwer zugänglich. Insbesondere diejenigen Bürgerwehren, die in rechtsextremistische Strukturen eingebunden sind und diejenigen, deren Mitglieder ein rechtsextremes Weltbild haben, sind gegenüber gesellschaftlichen Eliten, zu denen auch die Universität gehört, sehr skeptisch eingestellt. Hier zeigt sich das Spannungsfeld zwischen Forschungsinteresse und Forschungsethik. Es gilt, einen Zugang zum Forschungsgegenstand zu gewinnen, der die Bürgerwehren über das Vorhaben informiert, aber keine abschreckende Wirkung auf die Mitglieder hat.

Wie tritt die Wissenschaftlerin im Feld auf? Auf welche Art und Weise wird das Vorhaben der Studie erklärt? Diese Aspekte bedürfen einer Reflexion, um nicht aus Angst vor Zurückweisung durch die Bürgerwehren Informationen zurückzuhalten, die für eine informierte Zustimmung der Mitglieder der Bürgerwehren relevant sind. Im Folgenden wird daher die Rolle der Wissenschaftlerin sowohl im Prozess des Feldzugangs als auch im Feld selbst reflektiert (vgl hierzu auch die Kapitel 6.1.1, 7.1.1 und 8.1.1). Anschließend wird die Wahl einer ethnografischen Methodik für die Untersuchung von rechtsextremen Bürgerwehren kritisch geprüft. In Kapitel 4.1.2.3 werden schließlich die Maßnahmen des Datenschutzes dargelegt, die diese Studie vornimmt, um die Privatsphäre der Mitglieder der untersuchten Bürgerwehren zu schützen.

\subsubsection{Die Wissenschaftlerin im Feld}

Die Dynamik der Situation wird während der Untersuchung von der Anwesenheit und den Handlungen der Forscherin beeinflusst. Dieser Einfluss lässt sich nicht verhindern. Es geht auch nicht darum, ihn zu minimieren. Natürlich sollen Personen im Feld nicht aktiv dazu aufgefordert werden, Dinge zu tun, die sie ohne die Untersuchung nicht getan hätten. Aber es geht nicht darum, sich unsichtbar zu machen. Teilnahme bedeutet schließlich auch Sichtbarkeit in einem visuellen Sinne. Doch eine selbstverständliche Teilnahme kann gleichzeitig auch unsichtbarer machen. Unsichtbar in einem sozialen Sinne, wenn sich die Forscherin so selbstverständlich in die Situation einfügt, dass ihre Fremdheit in den Hintergrund tritt. 
»Kulturelle Felder verfügen über eine Eigenlogik, eine eigene Ordnung, die auch einen Beobachter, der sich treiben lässt, an die Hand nimmt und führt. $\ll^{43}$

Diese Eigenlogik gilt es zu erspüren und zu nutzen. Sich in sie einzufügen, ermöglicht ein Unsichtbar-werden in der Situation und erschließt der Forschenden die Logik der sozialen Interaktion der Teilnehmenden.

Natürlich macht es einen Unterschied, dass jemand Fremdes dabei ist und wer die beobachtende Person ist. Ihr Geschlecht, ihre soziale Herkunft, Alter, Aussehen, Habitus, all diese Merkmale beeinflussen die Beobachtung. Das ist nicht vermeidbar, sollte jedoch bei der Analyse der Daten berücksichtigt werden. Und: »Nicht jeder Ethnograf ist für jede Feldforschung geeignet. « ${ }^{44}$ Zum Beispiel kann eine weiblich gelesene Person Zugang zu sozialen Zusammenhängen bekommen, in denen nur Frauen agieren - während diese Räume Männern verschlossen bleiben. Gleiches gilt natürlich auch umgekehrt.

Ich - als Weiße, junge Frau mit universitärer Bildung - beeinflusse in meiner Rolle als Forschende die Situation und das Verhalten der Mitglieder der Bürgerwehr. Diese Rolle hat Vor- und Nachteile und lässt sich nicht leugnen oder minimieren. Ich nutze meine Rolle daher für diese Studie. Als junge blonde Frau ist es einfacher, einen guten Zugang zu einer männlich dominierten Gruppe zu bekommen, in der klassische Vorstellungen von Geschlechterrollen vorherrschen. Allein die Tatsache, dass ich mich für die Bürgerwehr interessiere, bedeutet Anerkennung für die Männer. Diesen Umstand gilt es zu nutzen. Denn gleichzeitig birgt die Anfrage auch großes Potential für ablehnende Reaktionen seitens der Bürgerwehren.

Die Kontaktaufnahme erfolgte im Falle der Bürgerwehr des Typs I über die Bürgermeisterin des Ortes, denn die Bürgerwehr ist im Ort gut integriert und anerkannt. Dass die Anfrage von einer Autoritätsperson übermittelt wurde, führte dazu, dass die Mitglieder Vertrauen in die Studie hatten. Bei den Bürgerwehren von Typ II und III erfolgte die Kontaktaufnahme über die persönlichen Profile einzelner Mitglieder auf Facebook, in erster Linie deshalb, weil sich Bürgerwehren und ihre einzelnen Mitglieder auf Facebook gut recherchieren lassen - auch dann, wenn die Bürgerwehr selbst nicht über eine eigene Facebookseite verfügt, so posten doch einzelne Mitglieder auf ihren 
privaten Profilen über ihr Engagement in der Bürgerwehr und sind auf diese Weise auffindbar. Auch die Wirkung, die ich als junge blonde Frau habe, lässt sich über Facebook besonders gut nutzen, um einen Zugang zu den Bürgerwehren zu bekommen. Durch eine Kontaktaufnahme über Facebook, wo sich viele Bürgerwehren organisieren, lässt sich leichter ein verbindlicheres Verhältnis aufbauen als beispielsweise durch eine Anfrage per E-Mail, weil beide Seiten die Fotos und Profile des jeweils anderen sehen.

Auch während der Beobachtung beeinflusst meine Person, mein Äußeres und was die Bürgerwehren in mir sehen die Beobachtungssituation. Ich versuchte, diese Projektionen auf meine Person zu nutzen, um ein Vertrauensverhältnis aufzubauen, in dem sich die Mitglieder der Bürgerwehren entspannt verhalten und sicher fühlen konnten. Auch bei den Antworten auf meine Fragen im Feld trägt meine Rolle dazu bei, dass ein Raum entsteht, indem sich die Mitglieder wohl fühlen und offen sprechen wollen.

Ich trete als Wissenschaftlerin auf, als Vertreterin einer gesellschaftlichen Institution, die Eliten produziert und die von Teilen der Bürgerwehren kritisch gesehen wird. Dennoch haben die Mitglieder keine Gefahr in mir gesehen. Hier wirkt meine Rolle als weiblich gelesene junge Frau in die entgegengesetzte Richtung. Hier bewirken sexistische Vorstellungen, dass die Macht, die ich als Vertreterin der gesellschaftlichen Elite zugeschrieben bekomme, weniger stark wirkt. So war das Verhältnis zu den Mitgliedern der Bürgerwehren eher freundschaftlich, und sie haben sich über mein Interesse gefreut. Gleichzeitig blieb allein aufgrund der Sichtbarkeit des Aufnahmegeräts meine Rolle als Wissenschaftlerin stets präsent.

Selbstverständlich werden die Auswirkungen meiner Person auf die Situation im Analyseprozess berücksichtigt. Denn jeder ethnografischen Forschung wohnt ein gewisses Herrschaftsverhältnis inne. Die Ethnografin beschreibt und deutet das Verhalten der Menschen, die sie beobachtet. Wenn die beobachtete Gesellschaft in einem historischen Hierarchieverhältnis zur Herkunftsgesellschaft der Ethnografin steht, so erwächst daraus eine besondere Verantwortung. Diesen Umstand verhandelte die Writing Culture-Debatte der 1980er Jahre.

Ich befinde mich mit meiner Feldforschung in Deutschland. Der kulturelle Kontext, die Sprache, die Narrative in der untersuchten sozialen Situation überschneiden sich in weiten Teilen mit denen, die ich verinnerlicht habe. Daher ist das Hierarchieverhältnis zumindest nicht von rassistischen und postkolonialen Strukturen geprägt. Dennoch bleibt ein Hierarchieverhältnis bezüglich des Bildungsstandes bestehen. Das ist zwar keineswegs bei allen 
Bürgerwehren gleich stark vorhanden - so haben einige Mitglieder der untersuchten Bürgerwehren ebenfalls studiert - dennoch gilt es, das Hierarchieverhältnis, das der Beobachtungssituation auf Grund einer Klassenzugehörigkeit innewohnt, bei der Analyse des Datenmaterials zu berücksichtigen.

\subsubsection{Mit Rechten reden: Überlegungen zur ethnografischen Untersuchung von Bürgerwehren}

Prinzip einer teilnehmenden Beobachtung ist die aktive Teilnahme am Geschehen. Für ein besseres Verständnis der sozialen Situation soll die Forscherin sich so weit wie möglich auf die Welt einlassen, die sie untersuchen will. Sie soll erfahren, was die Menschen erfahren, die sie begleitet und befragt, soll denken, wie sie denken. Dabei besteht die Gefahr, Dinge mitzutragen, die den Wertvorstellungen der Forscherin widersprechen. Dieser Umstand existiert bei jeder ethnografischen Forschung, es stellt sich jedoch die Frage, ob diese methodische Herangehensweise für eine Untersuchung von sozialen Situationen geeignet ist, in denen menschenverachtende Einstellungen vorhanden sind.

Bürgerwehren setzen ihre eigenen Vorstellungen von Recht um. Dabei agieren sie mit physischer Präsenz im öffentlichen Raum und wirken auf die Menschen, denen sie begegnen, selbst wenn sie nicht direkt mit ihnen interagieren. Sie schüchtern ein, verbreiten Angst. In einigen Fällen sind zudem gewalttätige Übergriffe auf Unbeteiligte dokumentiert. ${ }^{45}$ Läuft die Forscherin mit der Bürgerwehr mit, so trägt sie ihrer Legitimation bei. Sie unterstützt allein durch ihre Anwesenheit die physische Stärke, mit der die Bürgerwehr auftritt. In einem solchen Setting bleibt - unabhängig vom Forschungsauftrag - die ethische Verantwortlichkeit für das, was geschieht, bei der Forscherin. ${ }^{46}$ Sie muss eigene Grenzen formulieren und einschreiten, wenn Menschen zu Schaden kommen.

Dieser Aspekt ist besonders bei den Bürgerwehren von Typ II und III relevant. Hier besteht die größte Gefahr, dass es während der Patrouille zu ver-

45 Vgl. bspw. Stürzenhofecker, Michael: Angebliche Bürgerwehr greift Asylbewerber an. In: Zeit Online vom 2.06.2016. Online unter: https://www.zeit.de/gesellschaft/zeitg eschehen/2016-06/arnsdorf-sachsen-fluechtling-buergerwehr-supermarkt (Zugriff am 2.1.2021).

46 Vgl. Kaschuba, Wolfgang (2006): Einführung in die Europäische Ethnologie. München: Beck. S. 207. 
balen oder physischen Übergriffen kommt. ${ }^{47}$ Eine weitere Problematik bei einer teilnehmenden Beobachtung von Bürgerwehren trifft jedoch auf alle drei Typen zu: Die Methodik erfordert einen Perspektivwechsel. Die Forscherin soll sich ganz auf die Erfahrungswelt der zu beobachtenden Personen einlassen, ihre Sicht der Dinge nachvollziehen. ${ }^{48}$ Dabei besteht die Gefahr, rechte Denkweisen als legitime Erklärungsmuster anzuerkennen, anstatt sich klar von ihnen $\mathrm{zu}$ distanzieren und sie als menschenverachtend $\mathrm{zu}$ benennen.

In der Diskussion um kulturellen Relativismus in der ethnologischen Forschung ist es lauf Clifford Geertz wichtig, »[to place] morality beyond culture and knowledge beyond both. ${ }^{49}$ Moralische Werte sind demnach weder relativ, noch haben bestimmte Gesellschaften (etwa die westlichen) ein Monopol darauf, diese zu formulieren. Aus dieser Absage sowohl an einen moralischen Eurozentrismus als auch an kulturellen Relativismus heraus lässt sich Kritik an der untersuchten kulturellen Praxis formulieren. Diese Kritik muss jedoch jenseits von Projektionen erfolgen. Sie darf nicht auf Vorurteilen über die zu untersuchenden Subjekte beruhen und nicht auf einer Konstruktion des Fremden in Abgrenzung zur eigenen Gesellschaft erfolgen.

Die Diskussion um kulturellen Relativismus bezieht sich vor allem auf transkulturelle Forschungssituationen, in denen der/die Ethnograf* in in ein anderes Land fährt, eine ihm/ihr unbekannte Gesellschaft aufsucht. Aber auch im Falle der Bürgerwehren betritt die Forscherin ihr unbekannte lokale Strukturen. Zwar sind der gesamtgesellschaftliche Kontext und die Sprache vertraut, doch kennt sie weder die sozialen Dynamiken innerhalb der Bürgerwehr noch deren Position in der lokalen Bevölkerung. Ein »sprechen von « ist immer »ein sprechen für « ${ }^{50}$, egal wie vertraut die Forscherin mit dem von ihr untersuchten Raum ist. Die Hierarchie ethnologischer Forschungssituationen bleibt bestehen.

47 In Chemnitz beispielsweise sind Mitglieder einer Bürgerwehr angeklagt, weil sie auf einer Patrouille rassifizierte Menschen bedroht und verletzt haben sollen. VgI. SZOnline vom 15.09.2018: Mitglieder selbsternannter Bürgerwehr attackieren Migranten. Online unter: https://www.sueddeutsche.de/politik/chemnitz-mitglieder-selbstern annter-buergerwehr-attackieren-migranten-1.4131431 (Zugriff am 2.1.2021).

48 Vgl. Breidenstein, Georg et al. (2013): Ethnographie. Eine Praxis der Feldforschung. Konstanz: UVK. S. 18.

49 Geertz, Clifford (2000): Anti-anti-relativism. In: Ders: Available Light. Anthropological Reflections on Philosophical Topics. New Jersey: Princeton University Press. S. 65.

50 Vgl. Breidenstein, Georg et al. (2013): Ethnographie. Eine Praxis der Feldforschung. Konstanz: UVK. S. 19. 
Trotz dieser beiden wichtigen Einwände gegen eine ethnografische Betrachtung von Bürgerwehren in Deutschland bleibt die Ethnografie der am besten geeignete methodische Zugang zum Phänomen. Bürgerwehren sind ein derart flüchtiger und schwer zugänglicher Untersuchungsgegenstand, dass nur ein direkter Zugang Verständnis ermöglicht. Und auch, wenn die teilnehmende Beobachtung eine Verschmelzung mit dem Untersuchungsgegenstand erfordert, so geschieht diese nur bis zu einem gewissen Grad. Während teilgenommen wird, bleibt ein Teil von der Forschenden distanziert und erfasst, was vor sich geht. Ethnograf*innen nutzen dabei ihre eigene Fremdheit, um klarer zu sehen..$^{51}$

\subsubsection{Maßnahmen zur Wahrung der Persönlichkeitsrechte von Mitgliedern der Bürgerwehren}

Forschungsethisch ist neben dem informierten Einverständnis der Teilnehmenden außerdem die Wahrung ihrer Anonymität sowie die Vermeidung einer Schädigung der Einzelpersonen zentral. ${ }^{52}$ In dieser Studie werden daher die untersuchten Bürgerwehren pseudonymisiert. Das heißt, die Orte, an denen die Bürgerwehren patrouillieren sowie die Mitglieder der Bürgerwehren werden nicht namentlich genannt und darüber hinaus in ihrer Beschreibung so allgemein gehalten, dass die Beschreibung auf mehrere Orte, Bürgerwehren und Menschen zutreffen könnte.

Der Ort, an dem die Bürgerwehr des Typs III ihre Patrouillen abhält, wird jedoch in dieser Studie genannt. Die Entscheidung, auf eine Pseudonymisierung des Ortes der Bürgerwehr Berlin Mitte zu verzichten, erfolgte nach sorgsamer Abwägung. ${ }^{53}$ Berlin Mitte ist als Schauplatz für die Analyse der Bürgerwehr zentral. Es ist einer der Orte Deutschlands mit der größten Polizeipräsenz. Gleichzeitig hat er als Zentrum der Macht demokratischer Institutionen eine symbolische Wirkung, ohne deren Berücksichtigung die Analyse der Bürgerwehr in Berlin Mitte nicht gerecht werden würde. Zudem war nur ein Mitglied der Bürgerwehr Berlin Mitte bereit für ein Interview im Feld. Dieses Mitglied tritt gegenüber der Presse und in sozialen Medien mit vollem

\footnotetext{
51 Vgl. ebd., S. 8.

52 Vgl. Unger/Narimani/M'Bayo (2014): Forschungsethik in der qualitativen Forschung. Wiesbaden: VS Verlag. S. 47.

53 Zum Prozess der Abwägung von Schutzmaßnahmen für die Feldteilnehmenden vgl. Breidenstein, Georg et al. (2013): Ethnographie. Eine Praxis der Feldforschung. Konstanz: UVK. S. 69.
} 
Namen und Foto als Mitglied der Bürgerwehr auf und ist zudem als Mitglied des Berliner Landesverbands der NPD eine öffentliche Person. Die Wiedererkennbarkeit dieses Mitglieds der Bürgerwehr Berlin Mitte wird in dieser Studie in Kauf genommen, weil eine daraus erwachsende Schädigung für die Person nicht zu erwarten ist.

Allen bei den teilnehmenden Beobachtungen und den Feldinterviews anwesenden Mitgliedern der untersuchten Bürgerwehren wurde mitgeteilt, dass die Gespräche aufgezeichnet werden und die Studie als Doktorarbeit veröffentlicht wird. Das Transkript aller Interviews wird in dieser Studie nicht veröffentlicht, weil es Rückschlüsse auf die Identitäten der Mitglieder der Bürgerwehren zuließe. Es kann jedoch für Forschungszwecke auf Anfrage eingesehen werden.

\subsection{Empirisches Material: "Daten [sind] in Wirklichkeit unsere Auslegungen davon."}

Das empirische Material einer ethnografischen Forschung kann je nach Feld ein weites Spektrum umfassen (Dokumente, Aufzeichnungen und Protokolle der Ethnograf*innen, Transkript von Interviews, Skizzen, Fotos, Videoaufnahmen, Archivmaterialien, Zeitungsartikel, Internetseiten und Artefakte). ${ }^{54}$ Dabei gibt es keine reinen Daten. Die Daten werden nicht dort draußen gefunden, sondern sind abhängig von Auswahl, Beobachtung und Interpretation. ${ }^{55}$

Bei den in dieser Studie gewählten methodischen Herangehensweisen der teilnehmenden Beobachtung und der (Gruppen-)Interviews im Feld entstehen die aus der Feldforschung gewonnenen Daten erst durch die Forscherin selbst. Es gibt keine bestehenden Texte, die auf eine Analyse warten. Bei einer teilnehmenden Beobachtung schreibt die Forscherin ihre Beobachtungen auf. Was sie im Feld beobachtet, ist immer gefiltert durch ihren Blick auf die Situation. Erfahrungswissen ist erst einmal nicht datenförmig und muss erst durch die Forscherin in Datenmaterial verarbeitet werden. Es wird versprachlicht, das heißt, es müssen Wörter gefunden werden, die die Erfahrung

54 Vgl. Breidenstein, Georg et al. (2013): Ethnographie. Eine Praxis der Feldforschung. Konstanz: UVK. S. 115.

55 Vgl. ebd., S. 9. 
der Forscherin abbilden. Das Gesehene bleibt dabei nur ein kleiner Teil der (körperlichen) Erfahrung, denn die Erfahrung erfolgte mit allen Sinnen. ${ }^{56}$

Die Interviews liefern zwar transkribierte Aussagen der Interviewten, also Texte, die nicht von der Forscherin selbst formuliert wurden, doch wären auch diese Daten nicht entstanden, wenn die Forscherin nicht die Interviewsituation hergestellt hätte und während des Interviews fragend die Richtung der Aussagen mit beeinflusst hätte.

Im Falle der Typ III-Bürgerwehr fließen ergänzend Social-Media-Posts der Mitglieder in die Analyse mit ein. Diese Bürgerwehren haben aktivistische Beweggründe für ihre Gründung, sie nutzen die Struktur »Bürgerwehr« als Mittel politischer Gestaltung. Und bei ihren politischen Meinungen besteht die größte Abweichung zu einem demokratischen Konsens. Weil insbesondere bei den Bürgerwehren vom Typ III die Gefahr besteht, dass sie aus politischem Kalkül heraus einige ihrer Einstellungen zurückhalten, wird hier vereinzelt ergänzend auf Äußerungen in den sozialen Medien zurückgegriffen, um die politische Selbstverortung der Mitglieder zu verifizieren.

Im Folgenden wird zunächst Clifford Geertz' Ansatz einer dichten Beschreibung diskutiert, mit dem die Daten der teilnehmenden Beobachtung generiert werden sollen. Anschließend wird ein Leitfaden für die Gruppeninterviews entwickelt und das Codierungssystem für die Analyse von dichten Beschreibungen und Interviewtranskripten dargelegt.

\subsection{1 „Das 'Gesagte` [...] dem vergänglichen Augenblick entreißen. « - Dichte Beschreibung nach Clifford Geertz}

Der (Mit-)Initiator der Writing Culture-Debatte Clifford Geertz liefert 1987 in seinem Aufsatz »Dichte Beschreibung. Bemerkungen zu einer deutenden Theorie von Kultur einen Ansatz zur Gewinnung von Daten, der sich von dem lange in der Ethnologie vorherrschenden tagebuchartigen Festhalten von erlebten Situationen unterschied. Geertz fordert eine Reflexion über die Darstellungsweisen ethnografischer Forschung ${ }^{57}$ und formuliert mit der »dichten Beschreibung « eine neue Form der schriftlichen Darstellung von Feldforschungsergebnissen. Er betont die Subjektivität, die den Daten innewohnt, mit denen Sozialwissenschaftler*innen und Ethnolog*innen arbeiten: »Das

\footnotetext{
56 Vgl. ebd., S. 115.

57 Vgl. Geertz, Clifford (1987): Dichte Beschreibung. Frankfurt a.M.: Suhrkamp. S. 28f. Fußnote 3.
} 
was wir als unsere Daten bezeichnen, [sind] in Wirklichkeit unsere Auslegungen davon. ${ }^{58}$ Das ist unvermeidlich und auch nicht schlimm, doch dürfe man nicht den Eindruck erwecken, Forschung sei eine Sache der Beobachtung und weniger eine der Interpretation. Denn tatsächlich verhalte es sich umgekehrt. ${ }^{59}$

Der »dichten Beschreibung « liegt in Anlehnung an Max Weber ein semiotischer Kulturbegriff zugrunde. Also ein auf zeichenhafte Bedeutungen beruhender Kulturbegriff. Menschen sind demnach in ein "selbstgesponnenes Bedeutungsgewebe verstrickt «, und dieses Gewebe bezeichnet Geertz als Kultur. ${ }^{60}$ Damit distanziert er sich von Ansätzen, die versuchen, Kultur und Gesellschaft in ein einfaches Abbildungsverhältnis zu bringen, und vor allem von jener Vorstellung, die Nation und Kultur als Einheit begreift. ${ }^{61}$

»Als ineinandergreifende Systeme auslegbarer Zeichen (...) ist Kultur keine Instanz, der gesellschaftliche Ereignisse, Verhaltensweisen, Institutionen oder Prozesse kausal zugeordnet werden können. Sie ist ein Kontext, ein Rahmen, in dem sie verständlich - nämlich dicht - beschreibbar sind. ${ }^{62}$

Geertz fragt, "was macht der Ethnograph? Antwort: Er schreibt.« ${ }^{63}$ Dem Schreiben kommt bei einer ethnografischen Forschung eine besondere Bedeutung zu. Zwar werden auch andere Forschungsergebnisse schriftlich festgehalten, jedoch ist die Verschriftlichung bei einer Ethnografie die Übertragung des beobachteten Geschehens in die sprachliche Form. Während bei anderen methodischen Zugängen bereits schriftliche Quellen zur Verfügung stehen (Artikel, Dokumente, Interviewtranskripte), müssen bei der teilnehmenden Beobachtung für das Geschehen erst Worte gefunden werden. ${ }^{64}$ In diesem Findungsprozess liegt die eigentliche Arbeit der Forscherin. Und diesen Prozess des Worte-findens beschreibt Geertz in seinem Essay.

Kultur zu untersuchen kann also keine experimentelle Wissenschaft sein, die nach Gesetzen sucht. Sie muss interpretieren und nach Bedeutungen su-

\footnotetext{
58 Vgl. ebd., S. 14.

59 Vgl. ebd., S. 14.

60 Vgl. ebd., S. 9.

61 Vgl. Kaschuba, Wolfgang (2006): Einführung in die Europäische Ethnologie. München: Beck. S. 124.

62 Geertz, Clifford (1987): Dichte Beschreibung. Frankfurt a.M.: Suhrkamp. S. 21.

63 Ebd. S. 28.

64 Vgl. Breidenstein, Ceorg et al. (2013): Ethnographie. Eine Praxis der Feldforschung. Konstanz: UVK. S. 35.
} 
chen. ${ }^{65}$ Bloßes Beschreiben des Erlebten reicht nicht aus. Geertz unterscheidet zwischen einer »dichten « und einer »dünnen« Beschreibung sozialer Situationen. Während eine dünne Beschreibung oberflächlich das Gesehene versprachlicht, berücksichtigt eine dichte Beschreibung den sozialen Kontext, in dem Handlungen geschehen.

Um diesen Unterschied zu verdeutlichen, zitiert Geertz ein Beispiel des Philosophen Gilbert Ryle. ${ }^{66}$ In diesem heißt es in etwa: Wenn ein Junge einem anderen Jungen zuzwinkert, dann kann dieses Augenzwinkern als eine schnelle Bewegung des Augenlids beschrieben werden. Das wäre eine dünne Beschreibung der Handlung. Berücksichtigt man bei der Beschreibung jedoch den Kontext, so muss man die Handlung als eine Kommunikation zwischen den beiden Jungen beschreiben. Der Junge vollzieht seine Handlung absichtlich, richtet sie an jemand Bestimmten, um diesem eine Nachricht $\mathrm{zu}$ übermitteln. Wenn sich der Kontext ändert, wenn also zum Beispiel der zwinkernde Junge zuvor einen anderen Menschen zwinkern sah und sich nun mit seiner Augenlidbewegung parodierend auf diese frühere Begegnung bezieht, so muss auch die Beschreibung dieser Handlung dies berücksichtigen, sofern sie als eine »dichte Beschreibung« gelten will.

Im Falle der Beobachtung einer Bürgerwehr auf Patrouille sind die Bewegungen der Gruppe nicht einfach nur die von Bürger*innen, die sich im öffentlichen Raum bewegen, weil sie z.B. eine Runde spazieren gehen oder sich von A nach B bewegen - die Bewegungen der Bürgerwehr müssen gemäß ihrer Rolle als Bürgerwehr gelesen werden. So ist das Abbiegen in eine Seitenstraße eine Verfolgung, wenn zuvor eine von der Bürgerwehr für »verdächtig« gehaltene Person diesen Weg gewählt hat. Oder wenn die Bürgerwehr eine*n Passant" in anspricht und danach fragt, was er oder sie macht, so ist dies wahrscheinlich kein gewöhnlicher Plausch unter Nachbar*innen. Die Situation ist eine Machtdemonstration der Bürgerwehr. Sie treten als solche auf, stellen Fragen. Zwischen Passant*innen und den Mitgliedern der Bürgerwehr besteht ein Hierarchieverhältnis.

Das wird beispielsweise bei der teilnehmenden Beobachtung der Bürgerwehr von Typ III deutlich. Hier gehen die Männer in ihrer Rolle als Bürgerwehr am Hauptbahnhof auf Obdachlose zu, die die Straßenzeitung verkaufen. Alleine die zur Tür des Bahnhofsgebäudes weisende Armbewegung eines der Mitglieder der Bürgerwehr bewirkt, dass ein Junge, der mit einem

65 Vgl. Geertz, Clifford (1987): Dichte Beschreibung. Frankfurt a.M.: Suhrkamp. S. 9.

66 Vgl. Ryle, Gilbert (1971): Collected Papers. Vol. 2. Oxon: Routledge 2009. S. 494ff. 
Pappbecher Passant*innen nach Geld fragt, davonläuft. Die Armbewegung des Bürgerwehrmitglieds wird erst in seiner Bedeutung erfassbar, wenn man den sozialen Kontext, sein Auftreten als Teil der Bürgerwehr, berücksichtigt.

Um alle Ebenen dieser sozialen Situation zu erfassen, bedarf es also einer besonders dichten Beschreibung: In welcher Stimmlage wird die Person angesprochen? Wie ist die Körpersprache der Mitglieder der Bürgerwehr? Wie positionieren sie ihre Körper in Bezug zu der angetroffenen Person? Welche (nonverbale) Interaktion findet zwischen den einzelnen Mitgliedern der Bürgerwehr statt, die das Verhalten der Passant*in bewerten?

Zwischen dünner und dichter Beschreibung liegt eine "geschichtete Hierarchie bedeutungsvoller Strukturen «. Und in diesem Rahmen müssen Handlungen verstanden und interpretiert werden. ${ }^{67}$ Denn ohne diese Strukturen gäbe es die Handlungen faktisch nicht. Sie liefern den Anlass und den Grund für die Aktion. Eine »dichte Beschreibung«versucht die Perspektive des Handelnden einzunehmen, die Welt durch seine Augen zu sehen und seinen Blick auf die Welt abzubilden. Das ist das Ziel der Ethnologie: Eine »Erweiterung des menschlichen Diskursuniversums «. ${ }^{68}$

\subsubsection{Der Leitfaden für die Interviews mit den Bürgerwehren}

Die zweite Komponente des empirischen Materials wird durch Gruppeninterviews generiert. Der hierfür entwickelte Leitfaden versteht sich als ein Orientierungsmuster, das in erster Linie dazu dienen soll, die für die Forschung wichtigen Aspekte bei allen untersuchten Bürgerwehren abzufragen und nichts zu vergessen. Gleichwohl soll nicht starr an der Reihenfolge der Fragen festgehalten werden, um nicht vorhersehbare Reaktionen zu erfassen und darauf eingehen zu können. Die Interviewführung ist also ein »Prozess permanenter spontaner Operationalisierung «. ${ }^{69}$

\footnotetext{
67 Vgl. Geertz, Clifford (1987): Dichte Beschreibung. Frankfurt a.M.: Suhrkamp. S. 12.

68 Vgl. ebd., S. 20.

69 Hopf, Christel (1978): Die Pseudo-Exploration. Überlegungen zur Technik qualitativer Interviews in der Sozialforschung. In: Zeitschrift für Soziologie, Jg. 7, Heft 2, April 1978. S. 111.
} 


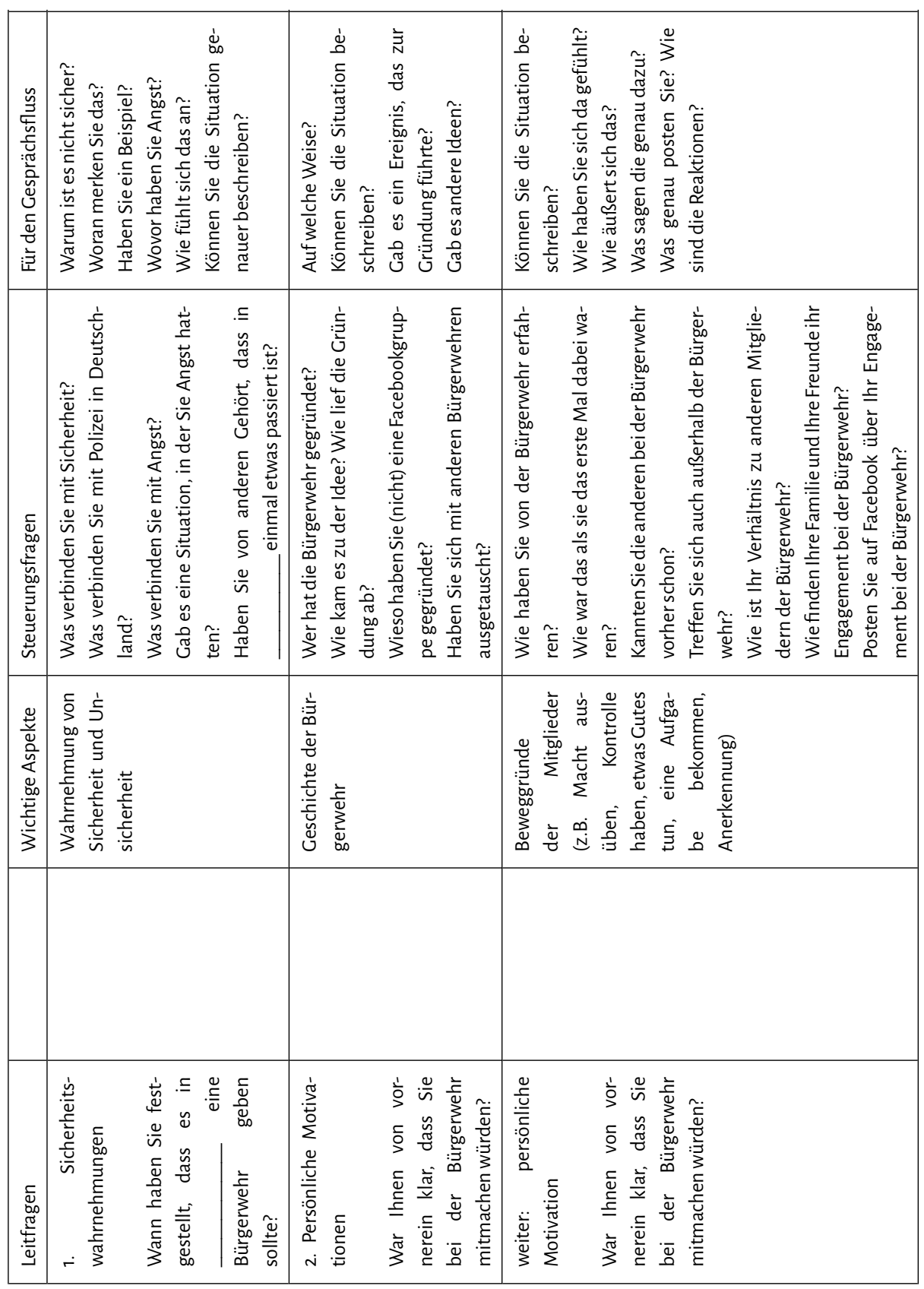




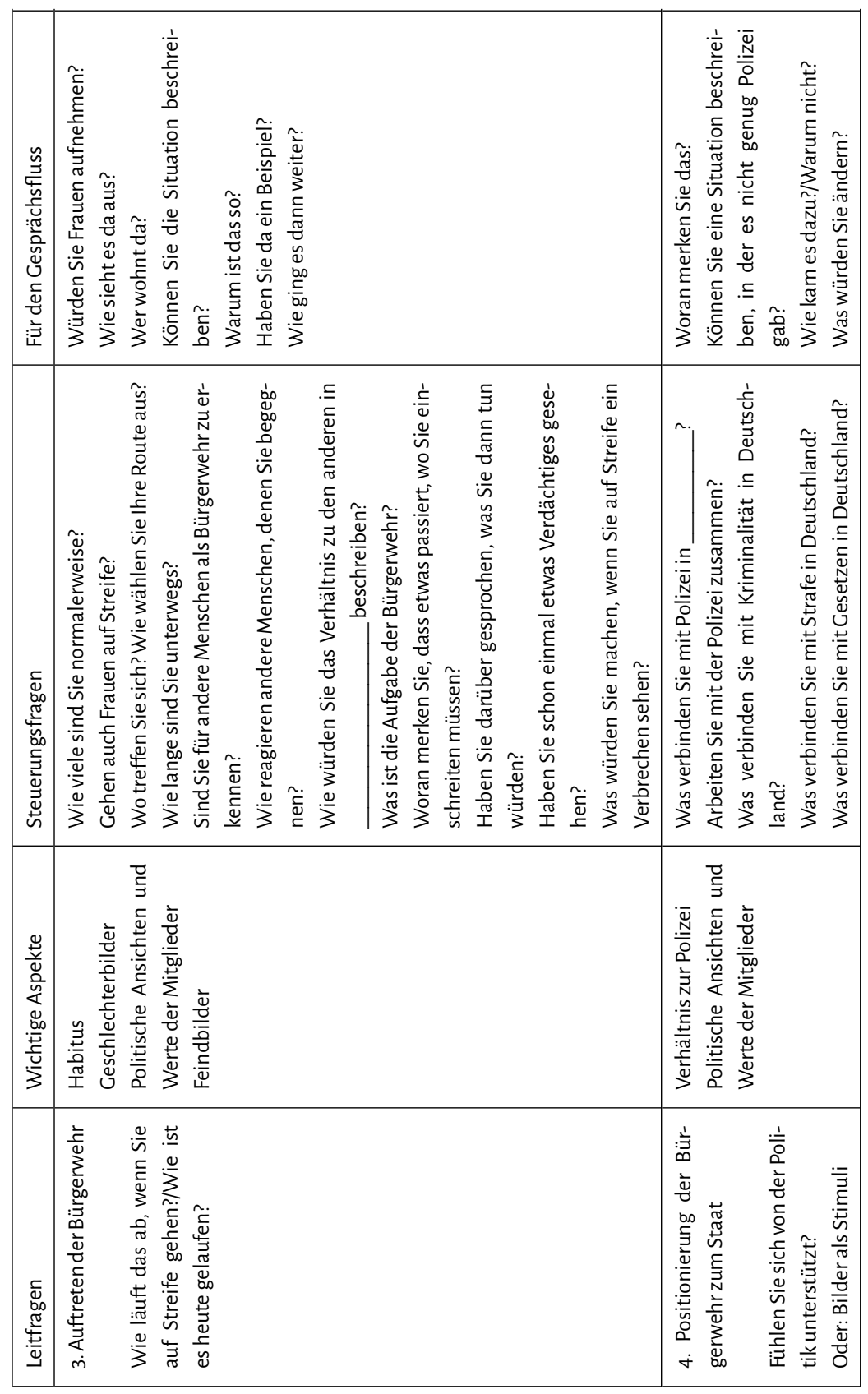




\subsubsection{Zum Vorgehen bei der Analyse des Datenmaterials}

Bei der Untersuchung ist es besonders wichtig, sich zunächst gänzlich auf die Perspektive der Mitglieder der Bürgerwehr einzulassen und diese durch eine dichte Beschreibung des Beobachteten abzubilden. Erst nach einem Distanzierungsprozess kann in der Analyse der gewonnenen Daten eine Einordnung des Phänomens Bürgerwehren als Versicherheitlichungsprozess sowie eine kritische Beurteilung erfolgen.

Mit dieser Herangehensweise reagiert Geertz auf die »Krise der Repräsentation«, die in den 1970er und 1980er Jahren in der ethnografischen Forschung diskutiert wurde und die die Kultur des Schreibens und Festschreibens in der Ethnologie und die damit verbundene Konstruktion von Kulturen thematisierte. Geertz verharrt nicht in der Lähmung der »Krise der Repräsentation«, er setzt ihr eine selbstbewusste Subjektivität entgegen. Bei der Untersuchung von Kultur sei es ebenso wenig wie in der Malerei möglich, eine Grenze zwischen Darstellung und zugrunde liegendem Inhalt $\mathrm{zu}$ ziehen. ${ }^{70}$

Die Forscherin besitzt eine interpretative Kompetenz, die Einzelbeobachtungen mikroskopischer Art mit makroskopischen Begebenheiten verschränkt. ${ }^{71}$ Dabei macht das Springen zwischen den Ebenen den Prozess des Interpretierens aus. Es setzt permanent Einzelbeobachtungen auf der Mikroebene in einen größeren Kontext. So werden die beobachteten Handlungen der Bürgerwehr im Kontext gesamtgesellschaftlicher Entwicklungen und politischer Debatten gesehen.

Wenn eine Person, die als nicht-deutsch wahrgenommen wird, von der Bürgerwehr angesprochen wird, so wird diese Handlung in den Rahmen der kulturellen Praktik des racial profiling eingebettet. Denn die Bürgerwehr bewegt sich hier mit ihrem Verhalten in einem gesellschaftlichen Kontext, ohne den ihr Verhalten nicht dicht beschrieben werden kann. So bedeutet ein Springen zwischen Mikro- und Makroebene für die Untersuchung der Bürgerwehren, dass ihr Verhalten nicht im luftleeren Raum geschieht. Um die Patrouille dicht beschreiben zu können, muss Verhalten auf der Mikroebene

\footnotetext{
70 Vgl. Geertz, Clifford (1987): Dichte Beschreibung. Frankfurt a.M.: Suhrkamp. S. 24.

71 Vgl. Meyer, Christian (2009): Ereignisethnographie und Methodologischer Situationalismus: Auswege aus der Krise der ethnographischen Repräsentation. In: Berger, Peter et al.: Feldforschung. Ethnologische Zugänge zu sozialen Wirklichkeiten. Berlin: Weißenseeverlag. S. 408.
} 
mit dem Wissen um kulturelle Praktiken beschrieben werden, die eine Manifestation gesellschaftlicher Debatten und politischer Ereignisse der Makroebene darstellen.

Die unsystematische Natur des Springens zwischen der Mikro- und der Makroebene führte zu der Kritik, die Daten seien in Geertz' Herangehensweise vorinterpretiert, die Forscherin scheine allwissend. ${ }^{72}$ Geertz' Standpunkt ist jedoch, dass jeglicher Beschreibung eine Subjektivität innewohnt. Der einzige Umgang mit dieser notwendigen Eigenschaft ethnografischer Forschung sei ein Sprung mitten rein in diese Probleme. ${ }^{73}$ Nur durch die explizite Berücksichtigung des Kontextes sozialer Handlungen könne Kultur überhaupt beschrieben werden. Ein vorgegebenes systematisches Springen würde dem jeweiligen Kontext nicht gerecht, die Struktur des Springens liegt vielmehr in der Verantwortung der interpretierenden Forscherin.

Wer Kultur untersuchen möchte, muss die soziale Wirklichkeit unterhalb und quer zur sprachlichen Ebene entschlüsseln. Akteure besitzen implizites Wissen, das sie nicht bewusst abrufen und nicht unbedingt versprachlichen können, Selbstverständlichkeiten, nonverbales Verhalten, Kleidung, nichtsprachliche Codes, Habitus, Lautäußerungen. Aufgabe der Forscherin ist es, diese Codes hervorzulocken, $\mathrm{zu}$ versachlichen und $\mathrm{zu}$ interpretieren.

Eine weitere Kritik an Geertz' Ansatz kam von Vincent Crapanzano. In seinem Beitrag zum Sammelband »Writing Culture« kritisiert er die Untersuchung, die Geertz mithilfe einer »dichten Beschreibung« vom balinesischen Hahnenkampf vorlegt. ${ }^{74}$ Geertz beschreibt den Hahnenkampf demnach nicht nur als Hahnenkampf, er beschreibt ihn als etwas, das über sich selbst hinaus weist, das Themen wie »Tod, Männlichkeit, Wut, Stolz, Verlust, Gnade und Glück « ${ }^{75}$ berührt und uns etwas über die balinesische Kultur an sich erzählt. ${ }^{76}$ Geertz' Beschreibung des balinesischen Hahnenkampfes sei somit »the constructed understanding of the constructed native's constructed point

Vgl. Meyer, Christian (2009): Ereignisethnographie und Methodologischer Situationalismus: Auswege aus der Krise der ethnographischen Repräsentation. In: Berger, Peter et al.: Feldforschung. Ethnologische Zugänge zu sozialen Wirklichkeiten. Berlin: Weißenseeverlag. S. $407 f$.

73 Ceertz, Clifford (1987): Dichte Beschreibung. Frankfurt a.M.: Suhrkamp. S. 43.

74 Vgl. Crapanzano, Vincent (1986): Hermes' Dilemma: The Making of Buversion in Ethnographic Description. In: Clifford, James (Hg.): Writing Culture. Berkeley: University of California Press. S. 51-76. 
of view. « ${ }^{77}$ Anders formuliert, der Versuch, den Standpunkt der beobachteten Subjekte einzunehmen und somit der »Krise der Repräsentation « zu entkommen, scheitere, weil Geertz sich anmaße, das Beobachtete in einen größeren Kontext zu setzen.

Doch vermag Geertz gerade durch das Springen zwischen Mikro- und Makroebene die Gefahr zu umgehen, sich im Kleinen zu verlieren und durch detailgetreue Beschreibung einer sozialen Situation das große Ganze aus dem Blick zu verlieren. Ohne den Bezug zur Makroebene brächte die Forschung keine Erkenntnis. Gleichzeitig ist das Ziel von Geertz' dichter Beschreibung keine essentialistische Beschreibung einer Kultur oder eines sozialen Raumes, es geht nicht um Vorhersagen, sondern lediglich um Generalisierungen in Bezug auf einen Einzelfall.

Was ist bei der Analyse von Datenmaterial aus einer dichten Beschreibung genau zu tun? Ziel ist, einen Zugang zur Gedankenwelt der Personen in der zu untersuchenden sozialen Situation zu bekommen, sodass »wir - in einem weiteren Sinne des Wortes - ein Gespräch mit ihnen führen können. $\aleph^{78}$ Die Aufgabe besteht konkret darin, die Vorstellungsstrukturen aufzudecken, die das Handeln der Personen in der sozialen Situation bestimmen. Dabei gilt es, ein analytisches Begriffssystem zu entwickeln, das diese Strukturen beschreiben kann. ${ }^{79}$ Ryle nennt diese Strukturen Codes. Geertz hingegen vergleicht die Analyse mit »dem Versuch, ein Manuskript zu lesen (im Sinne von reine Lesart entwickeln`), das fremdartig, verblasst, unvollständig, voll von Widersprüchen, fragwürdigen Verbesserungen und tendenziösen Kommentaren ist, aber nicht in konventionellen Lautzeichen, sondern in vergänglichen Beispielen geformten Verhaltens geschrieben ist. $\aleph^{80}$

Die Unterscheidung der »experimentellen Wissenschaften « zwischen Beschreibung und Erklärung bleibt auch bei Geertz' Ansatz erhalten. Sie liegt in einer »Unterscheidung zwischen $>$ Niederschrift< (>dichte Beschreibungs) und 'Spezifizierung< ((Diagnoser) ${ }^{81}{ }^{81}$ In der Analyse der durch eine »dichte Beschreibung" generierten Daten werden diese in einen gesellschaftstheoreti-

77 Vgl. Crapanzano, Vincent (1986): Hermes ¿ Dilemma: The Making of Buversion in Ethnographic Description. In: Clifford, James (Hg.): Writing Culture. Berkeley: University of California Press. S. 74.

78 Vgl. Geertz, Clifford (1987): Dichte Beschreibung. Frankfurt a.M.: Suhrkamp. S. 35.

79 Vgl. ebd., S. 39.

80 Vgl. ebd., S. 15.

81 Vgl. ebd., S. 39. 
schen Rahmen eingebettet. Geertz nennt hier unter anderem die Analysekategorien Nationalismus, Gewalt und Identität. ${ }^{82}$

Die Interpretationen finden auf einem höheren Abstraktionsniveau statt, sie können die ethnografischen Beschreibungen hinterfragen, sich von ihnen distanzieren. ${ }^{83}$ Mehr noch, die Forscherin sollte sich im Analyseprozess von den von ihr selbst generierten Daten überraschen lassen. Das mag unrealistisch klingen, meint aber vor allem, sich an die Überraschungen zu erinnern, die während der Datenerhebung entstanden sind und Widersprüche zu bemerken, die erst bei der Revision des Geschriebenen auffallen. Zudem ist es möglich, sich durch die Einnahme eines exotisierenden Blickwinkels, der Vertrautes fremd erscheinen lässt, Überraschungen herauszuarbeiten. ${ }^{84}$

Die Bürgerwehren dieser Studie werden in diesem zweiten Schritt im Analyserahmen Versicherheitlichung (vgl. Kapitel 2) betrachtet. Der Versicherheitlichungsprozess, verstanden als eine kommunikative Handlung (performative action), zeigt sich sowohl im Moment der Patrouille als auch durch die Argumentationen und Deutungen, die die Mitglieder in den Interviews für ihr Engagement in der Bürgerwehr anbringen. Hier gilt es herauszuarbeiten, welche Themen als existential threats fungieren. Auch hier ist, um zu verstehen, wie diese existential threats politisiert wurden, ein Springen zwischen der Mikroebene (Verhalten und Äußerungen der Bürgerwehr) und der Makroebene (gesellschaftlicher Kontext) unabdingbar.

Bei der Analyse des Datenmaterials, der dicht beschriebenen Beobachtungen, werden gleichwohl keine Verallgemeinerungen oder Vorhersagen angestrebt, sondern lediglich Generalisierungen im Rahmen eines Einzelfalls. ${ }^{85}$ Die Forscherin deckt das »Gesagte« des sozialen Diskurses auf und entwickelt ein analytisches Begriffssystem, das geeignet ist, die Strukturen zu beschreiben, die das Handeln der Subjekte ermöglichen. ${ }^{86}$

82 Vgl. Ceertz, Clifford (1987): Dichte Beschreibung. Frankfurt a.M.: Suhrkamp. S. 43.

83 Vgl. Breidenstein, Georg et al. (2013): Ethnographie. Eine Praxis der Feldforschung. Konstanz: UVK.S. 120.

84 Vgl. ebd., S. 121.

85 Vgl. Geertz, Clifford (1987): Dichte Beschreibung. Frankfurt a.M.: Suhrkamp. S. 37.

86 Vgl. ebd. S. 39. 


\subsubsection{Die chronologische Struktur durchbrechen: Codierung des Datenmaterials}

Um einen methodisch sauberen Zugang zum Datenmaterial zu bekommen und die Analyse nachvollziehbar zu gestalten, wird das Datenmaterial anhand von Codes strukturiert. Die Codes werden anschließend den für die Analyse relevanten Kategorien zugeordnet, um die einzelnen Bedeutungsebenen zusammen zu denken und auf eine höhere Analyseebene zu ziehen. Das Codesystem bricht die chronologische Ordnung des Datenmaterials auf und ermöglicht eine fragestellungsgeleitete Strukturierung. Im Hintergrund laufen dabei der Analyserahmen Versicherheitlichung sowie die Untersuchungshypothesen mit.

Die Codes unterscheiden sich je nach Einzelfallstudie, weil nicht bei allen Typen die gleichen Motivationen für die Gründung der Bürgerwehr vorhanden sind und nicht die gleichen Mechanismen der Realitätswahrnehmung. Auch spielen in der Umsetzung der Patrouillen unterschiedliche bewusste und unbewusste Einflussfaktoren eine Rolle. Die Kategorien der Analyse der drei Einzelfallstudien sind jedoch in Teilen identisch, einerseits, um eine Vergleichbarkeit der drei Fälle zu ermöglichen, andererseits, weil alle drei Einzelfallstudien in Bezug zur gleichen Fragestellung stehen, unter demselben Analyserahmen analysiert werden und sich an den gleichen Untersuchungshypothesen orientieren.

Ein volles Verständnis der Codierungsstruktur ergibt sich erst in Zusammenhang mit den Analysen der Einzelfälle (vgl. Kapitel 6, 7 und 8), dennoch sei hier bereits ein wenig vorgegriffen und die Codierung des Datenmaterials erklärt. Dafür sind in der folgenden Tabelle die unterschiedlichen Codes der drei Bürgerwehren einander gegenübergestellt und ihre Zuordnung zu den Kategorien der Analyse aufgezeigt. 


\begin{tabular}{|c|c|c|c|}
\hline Kategorie & Typ I & Typ II & Typ III \\
\hline >Legitimation & $\begin{array}{l}\text { Codes: } \\
\text { >Eigentum } \\
\text { >Gefahr } \\
\text { >Polizei< } \\
\text { >Recht und } \\
\text { Ordnung }\end{array}$ & $\begin{array}{l}\text { Codes: } \\
\text { >Fremde< } \\
\text { >Gefahr< } \\
\text { >Polizei< } \\
\text { >Legalität< }\end{array}$ & $\begin{array}{l}\text { Codes: } \\
\text { >Eigentum } \\
\text { Gefahr } \\
\text { >Polizeiく } \\
\text { >Recht und } \\
\text { Ordnung }\end{array}$ \\
\hline $\begin{array}{l}\text { >Persönliche } \\
\text { Motivation< }\end{array}$ & $\begin{array}{l}\text { Unterklasse: } \\
\text { >Anerkennung` } \\
\text { Codes: } \\
\text { >Kompetenz‘ } \\
\text { >audience` } \\
\text { >Hilfsmittel } \\
\text { Unterklasse: } \\
\text { >Status` } \\
\text { Codes: } \\
\text { >Benachteiligungく } \\
\text { >Privilegien` }\end{array}$ & $\begin{array}{l}\text { Unterklasse } \\
\text { >Abstiegsangst‘ } \\
\text { Codes: } \\
\text { >Benachteiligungく } \\
\text { >Privilegien` } \\
\text { >Staat/System }\end{array}$ & $\begin{array}{l}\text { Codes: } \\
\text { >Macht‘ } \\
\text { >Männlichkeit‘ } \\
\text { >Gewalt‘ } \\
\text { >Hierarchie' }\end{array}$ \\
\hline >Habitus & $\begin{array}{l}\text { Codes: } \\
\text { >Macht` } \\
\text { >Männlichkeit } \\
\text { >Hierarchieく }\end{array}$ & $\begin{array}{l}\text { Codes: } \\
\text { >Männlichkeitく } \\
\text { >Kompetenz‘ } \\
\text { >audience` } \\
\text { >Hilfsmittel }\end{array}$ & \\
\hline $\begin{array}{l}\text { Kategorie } \\
\text { strategie }\end{array}$ & & & $\begin{array}{l}\text { Codes: } \\
\text { >Legalität< } \\
\text { >Macht< } \\
\text { >audience }\end{array}$ \\
\hline $\begin{array}{l}\text { spolitische } \\
\text { Positionen }\end{array}$ & $\begin{array}{l}\text { Codes: } \\
\text { >Fremde` } \\
\text { >Staatく } \\
\text { >Legalität< }\end{array}$ & $\begin{array}{l}\text { Codes: } \\
\text { >Rassismus } \\
\text { >Macht } \\
\text { >Gewalt< }\end{array}$ & $\begin{array}{l}\text { Codes: } \\
\text { >Rassismus } \\
\text { >Fremde } \\
\text { >Privilegienく }\end{array}$ \\
\hline
\end{tabular}

Die Kategorie >Legitimation existiert bei allen drei Einzelfallstudien, sie beinhaltet aber beispielsweise für Typ I und III den Code `Eigentum<, weil das für die Bürgerwehren das Referenzobjekt darstellt, das es zu schützen gilt. Die Bürgerwehr des Typs II benennt eine andere Bedrohungslage, sodass hier stattdessen der Code >Frauen $<$ auftaucht. 
Einige Codes sind je nach Fall unterschiedlichen Kategorien zugeordnet. So befindet sich beispielsweise der Code >Legalität bei Typ I in der Kategorie >politische Positionen<, weil die starke Bedeutung, welche die Bürgerwehr ihrer eigenen Einhaltung der geltenden Gesetze beimisst, veranschaulicht, welche politischen Positionen die Mitglieder teilen. Bei der Bürgerwehr des Typs III hingegen ist der Code >Legalität der Kategorie >Strategie zugeordnet, weil die Ausführungen zum Handeln als Bürgerwehr in Anbetracht der Gesetzeslage Teil einer Strategie der Bürgerwehr sind, den öffentlichen Raum $\mathrm{zu}$ kontrollieren.

Es gibt aber nicht nur Kategorien, die je nach Bürgerwehr unterschiedliche Codes enthalten, sondern auch solche, die nicht in gleicher Form bei allen drei Einzelfällen vorhanden sind. Die Kategorie spersönliche Motivation beispielsweise unterscheidet sich sehr zwischen den drei untersuchten Bürgerwehren, weil die individuellen Motivationslagen voneinander abweichen. Anstatt der Kategorie >Habitus< ist bei der Bürgerwehr des Typs III die Kategorie `Strategie< vorhanden. Das liegt daran, dass sich der Habitus der Typ III-Bürgerwehr so sehr an der politischen Strategie der Bürgerwehr orientiert, dass diese anstatt des Habitus' zur übergeordneten Analysekategorie erhoben wurde.

\subsection{Zusammenfassung der Methodik}

Die qualitative Studie untersucht exemplarisch drei Bürgerwehren, jeweils eine der drei Typen, die in Kapitel 3 hergeleitet wurden: Institutionalisierte Bürgerwehren, Autonome Bürgerwehren und Aktivistische Bürgerwehren. Weil sich die Charakteristika der einzelnen Gruppen insbesondere im Auftreten der Bürgerwehren im öffentlichen Raum manifestieren, wird für die Untersuchung eine ethnografische Methodik gewählt. Datenmaterial wird aus einer teilnehmenden Beobachtung und (Gruppen-)Interviews mit den Mitgliedern der ausgewählten Bürgerwehren im Feld gewonnen. Je nach Beschaffenheit des Feldes handelt es sich um Einzel- oder Gruppeninterviews.

Die teilnehmende Beobachtung generiert Daten über das Auftreten der Bürgerwehr im öffentlichen Raum. Sie ermöglicht eine Analyse des Habitus', den die Mitglieder in ihrer Rolle als Bürgerwehr an den Tag legen, und ihrer Interaktion mit Passant*innen sowie der Interaktionen untereinander. Im Anschluss an die Begleitung werden die Beobachtungen nach Clifford Geertz »dicht« beschrieben und nach einem Distanzierungsprozess analysiert. 
In den (Gruppen-)Interviews hingegen liegt der Fokus auf den Deutungen und Argumentationen der Mitglieder der Bürgerwehren: Welche Narrative haben sie für ihr Engagement? Was bedeutet für sie Sicherheit? Ergänzt werden die Daten über die politische Selbstverortung der Bürgerwehr im Falle der Typ III-Bürgerwehr durch Social-Media-Posts. Auf diese Weise wird sichergestellt, dass politische Meinungen, die aus Opportunitätsgründen zurückgehalten wurden, Eingang in die Untersuchung finden.

Das Datenmaterial aus dichten Beschreibungen der Beobachtungen sowie der Transkripte der Interviews wird für eine strukturierte und hypothesengeleitete Analyse codiert (siehe Tabelle in Kapitel 4.2.3.1). Zu Kategorien zusammengefasst, ermöglichen die Codes ein Aufbrechen der chronologischen Ordnung des Datenmaterials, sodass eine von Fragestellung und Analyserahmen strukturierte Analyse erfolgen kann. Bei der Analyse wird stets die soziale Position der Forscherin reflektiert, denn ihre Rolle als Weiße junge Frau, die als Vertreterin einer Bildungsinstitution auftritt, die gesellschaftliche Eliten produziert, wirkt in der Interviewsituation sowie während der teilnehmenden Beobachtung.

Im Folgenden wird nun in Kapitel 5 ein Überblick über die Bürgerwehren in Deutschland gegeben. Die drei Typen werden präziser beschrieben und voneinander angegrenzt, und die Fallauswahl dieser Studie wird erläutert. 
